November 19, 2013

\title{
Higgs windows to new physics through $d=6$ operators: Constraints and one-loop anomalous dimensions
}

\author{
J. Elias-Miró ${ }^{a, b}$, J.R. Espinosa ${ }^{b, c}$, E. Masso ${ }^{a, b}$, A. Pomarol $^{a}$ \\ a Dept. de Física, Universitat Autònoma de Barcelona, 08193 Bellaterra, Barcelona \\ b IFAE, Universitat Autònoma de Barcelona, 08193 Bellaterra, Barcelona \\ c ICREA, Institució Catalana de Recerca i Estudis Avançats, Barcelona, Spain
}

\begin{abstract}
The leading contributions from heavy new physics to Higgs processes can be captured in a model-independent way by dimension-six operators in an effective Lagrangian approach. We present a complete analysis of how these contributions affect Higgs couplings. Under certain well-motivated assumptions, we find that $8 \mathrm{CP}$-even plus $3 \mathrm{CP}$-odd Wilson coefficients parametrize the main impact in Higgs physics, as all other coefficients are constrained by non-Higgs SM measurements. We calculate the most relevant anomalous dimensions for these Wilson coefficients, which describe operator mixing from the heavy scale down to the electroweak scale. This allows us to find the leading-log corrections to the predictions for the Higgs couplings in specific models, such as the MSSM or composite Higgs, which we find to be significant in certain cases.
\end{abstract}




\section{Introduction}

The resonance observed at around $125 \mathrm{GeV}$ at the LHC [1] has properties consistent with the Standard Model Higgs boson. More precise measurements of its couplings will hopefully provide information on the origin of electroweak symmetry breaking (EWSB) in the Standard Model (SM). All natural mechanisms proposed for EWSB introduce new physics at some scale $\Lambda$, not far from the $\mathrm{TeV}$ scale, that generates deviations in the SM Higgs physics. From this perspective, a convenient framework for a model-independent analysis for these deviations is the effective Lagrangian approach that consists in enlarging the SM Lagrangian by including higher-dimensional operators built of SM fields [2]. These operators are the low-energy remnants of the heavy new physics integrated out at the scale $\Lambda$, which appears then as the scale suppressing these operators.

In this article we perform a complete study of the impact of the (dominant) dimension-six operators in the most important Higgs couplings. In particular, we calculate the corrections to single Higgs couplings, relevant for the main Higgs decays and production mechanisms. We will show that, for one family, there are $8 \mathrm{CP}$-even operators that can only affect Higgs physics and no other SM processes (at tree-level). This corresponds to the number of independent dimension-six operators that can be constructed with $|H|^{2}$, and implies that Higgs couplings to fermions, photons, gluons, and $Z \gamma$ (for which large corrections are still possible) are characterised by independent Wilson coefficients. The rest of operators that could in principle affect Higgs physics at tree-level also enter in other SM processes and therefore can be constrained by independent (non-Higgs) experiments. In our paper we will present the main experimental constraints on these operators, with a full dedicated analysis to be reported in Ref. [3]. Our article aims to complete part of the analysis of Higgs physics given in Refs. $[4,5]$.

Out of the $8 \mathrm{CP}$-even operators that only affect Higgs physics, 5 of them are "tree-level" operators and 3 are "one-loop", as we will explain. The 5 tree-level operators affect directly the Higgs couplings to fermions, the kinetic term of the Higgs and the Higgs self-couplings. We calculate the anomalous dimensions of these 5 operators, which allow us to describe the renormalization group ( $\mathrm{RG}$ ) evolution of these Wilson coefficients from the heavy scale $\Lambda$, where they are generated, down to the electroweak-scale. ${ }^{1}$ We apply these results to find the leading-log corrections to the predictions for Higgs-couplings in several Beyond the Standard Model (BSM) scenarios: the Minimal Supersymmetric Standard Model (MSSM), universal theories (such as composite-Higgs models) and models with a non-standard top. We find that the corrections from this running can be sizable for $\Lambda \sim$ few $\mathrm{TeV}$, and will become more relevant as we have better measurements of the Higgs couplings. We also calculate the anomalous dimensions of the operators contributing to the $S$ and $T$ parameters and to the $Z b \bar{b}$ couplings. The stringent experimental constraints on these quantities can then be translated into indirect bounds on Higgs operators.

\footnotetext{
${ }^{1}$ For the other 3 one-loop CP-even operators, as well as for the 3 one-loop CP-odd, the calculation of the main anomalous dimensions has been given in $[6,7]$.
} 
The article is organized as follows. In Section 2 we review the classification of operators into two classes: tree-level and loop-induced operators. We also discuss the related issue of the election of the basis of effective dimension-six $(d=6)$ operators. The modifications of the Higgs couplings as a function of the Wilson coefficients are presented in Section 3. Some of these Wilson coefficients can only be tested in Higgs physics, while the others are constrained by LEP and Tevatron. In Section 4 we show how to obtain such constraints. In Section 5 we calculate the renormalization group equations (RGE) of the Wilson coefficients most relevant for Higgs physics. In Section 6 we illustrate the impact of such RG effects on particular scenarios of interest. After this, we present our conclusions while some technical details are left for the Apendices. Appendix A deals with redundant operators and the field redefinitions that can be used to remove them from the Lagrangian. Appendix B presents the results for the one-loop anomalous dimensions of the Wilson coefficients before removing the redundant operators. Finally, Appendix $\mathrm{C}$ discusses the transformation properties of the $d=6$ operators under the custodial $S U(2)_{L} \otimes S U(2)_{R}$ symmetry, which can be helpful in understanding some of their properties (e.g., concerning their mixing under RG evolution).

\section{Dimension-six operator basis}

Let us consider a BSM sector characterized by a new mass-scale $\Lambda$ much larger than the electroweak scale $M_{W}$. We will assume, among other requirements to be specified later, that this sector preserves lepton and baryon number. By integrating out this sector and performing an expansion of SM fields and their derivatives $D_{\mu}$ over $\Lambda$, we obtain an effective Lagrangian made of local operators:

$$
\mathcal{L}_{\text {eff }}=\frac{\Lambda^{4}}{g_{*}^{2}} \mathcal{L}\left(\frac{D_{\mu}}{\Lambda}, \frac{g_{H} H}{\Lambda}, \frac{g_{f_{L, R}} f_{L, R}}{\Lambda^{3 / 2}}, \frac{g F_{\mu \nu}}{\Lambda^{2}}\right) \simeq \mathcal{L}_{4}+\mathcal{L}_{6}+\cdots
$$

where $\mathcal{L}_{d}$ denotes the term in the expansion made of operators of dimension $d$. By $g_{*}$ we denote a generic coupling, while $g_{H}$ and $g_{f_{L, R}}$ are respectively accounting for the couplings of the Higgs-doublet $H$ and SM fermions $f_{L, R}$ to the BSM sector, and $g$ and $F_{\mu \nu}$ represent respectively the SM gauge couplings and field-strengths. ${ }^{2}$ The Lagrangian in Eq. (1) is based on dimensional analysis and the dependence on the couplings is easily obtained when the Planck constant $\hbar$ is put back in place. All couplings introduced in Eq. (1) can be useful as bookkeeping parameters. In particular, a term in the Lagrangian that contains $n$ fields, will carry some coupling to the power $n-2$ (in this counting, $\lambda$, the Higgs quartic-coupling, is formally of order $g_{*}^{2}$ ).

The dominant effects of the BSM sector are encoded in $\mathcal{L}_{6}$, as $\mathcal{L}_{4}$ leads only to an unphysical redefinition of the SM couplings. There are different bases used in the literature for the set of independent $d=6$ operators in $\mathcal{L}_{6}$. Although physics is independent of the choice of

\footnotetext{
${ }^{2}$ With this we are assuming that the SM gauge symmetry is also realized at energies above $\Lambda$ and therefore the couplings of the gauge bosons to the BSM sector are the SM gauge couplings. We can relax this assumption by replacing $g$ by an arbitrary coupling.
} 
basis, it is clear that some bases are better suited than others in order to extract the relevant information e.g. for Higgs physics. A convenient feature to ask of a good basis is that it captures in few operators the impact of different new-physics scenarios, at least for the most interesting cases. For example, in universal theories, defined as those BSM scenarios whose corrections can be encoded in operators made only of SM bosons, the bases used in Refs. [4,8] are appropriate since the physics effects can be captured by just $14 \mathrm{CP}$-even $d=6$ operators. Therefore, 14 is the number of independent parameters of the new physics effects and this number must be the same in all bases. However, the list of operators required to describe this same physics can contain many more than 14 operators in other bases, as for example in that of Ref. [9]. It follows that if we use such alternative bases to study universal theories there will be correlations among operator coefficients, making the analysis more cumbersome.

Another important consideration for the choice of basis is to separate operators whose coefficients are expected to have different sizes (again, at least in the main theories of interest). For example, it is convenient to keep separated the operators that can be induced at tree-level from integrating weakly-coupled states from those that can only be generated at the one-loop level. This helps in determining the most relevant operators when dealing with a large class of BSM scenarios such as supersymmetric, composite Higgs or little Higgs models among others. As shown in Ref. [7], this criterium is also useful when considering one-loop operator mixing, since one finds that tree-level induced operators often do not contribute to the RGE flow of one-loop induced ones, independently, of course, of the UV origin of the operators. In this particular sense, the basis of [7] is better suited than that of [8]. It is obvious that to meet all the criteria given above we do not need to sacrifice generality (a main goal of this article), as long as one keeps a complete basis of operators, as we do.

The operators of our basis will be broadly classified in three classes $[4,7]$. The first two classes will consist of operators that could in principle be generated at tree-level when integrating out heavy states with spin $\leq 1$ in renormalizable weakly-interacting theories. As we show in Appendix A, these operators can be written as products of scalar, fermion or vector currents of dimension less than $3 .^{3}$ Among these current-current operators we call operators of the first class those that involve extra powers of Higgs fields or SM fermions. They will be proportional to some power of the couplings $g_{H}$ or $g_{f_{L, R}}$, respectively. The importance of the operators of the first class is that they can be the most sizeable ones when the theory is close to the strong-coupling limit, $g_{H}, g_{f_{L, R}} \sim 4 \pi$. Operators of the second class are instead those that involve extra (covariant) derivatives or gauge-field strengths and, according to Eq. (1), are generically suppressed by $1 / \Lambda^{2}$ times a certain power of gauge couplings. Finally, in the third class, we will have operators that cannot be generated from a tree-level exchange of heavy fields and can only be induced, in renormalizable weakly-coupled theories, at the one-loop level. In this case, we expect these operators to be suppressed by $g_{*}^{2} /\left(16 \pi^{2} \Lambda^{2}\right)$.

\footnotetext{
${ }^{3}$ This, together with the fact that field-redefinitions through equations of motion do not mix the two types of operators, makes the classification well defined and unambiguous.
} 
We then classify the $d=6$ operators as

$$
\mathcal{L}_{6}=\sum_{i_{1}} g_{*}^{2} \frac{c_{i_{1}}}{\Lambda^{2}} \mathcal{O}_{i_{1}}+\sum_{i_{2}} \frac{c_{i_{2}}}{\Lambda^{2}} \mathcal{O}_{i_{2}}+\sum_{i_{3}} \frac{\kappa_{i_{3}}}{\Lambda^{2}} \mathcal{O}_{i_{3}},
$$

where, for notational convenience, we introduce the one-loop suppressed coefficients

$$
\kappa_{i_{3}} \equiv \frac{g_{*}^{2}}{16 \pi^{2}} c_{i_{3}}
$$

for the third class of operators. In weakly-coupled theories, $c_{i} \sim f_{i}\left(g / g_{*}, g_{H} / g_{*}, \ldots\right)$, where $f_{i}\left(g / g_{*}, g_{H} / g_{*}, \ldots\right)$ are functions that depend on ratios of couplings. We refer to the operators $\mathcal{O}_{i_{1}}$ and $\mathcal{O}_{i_{2}}$ as "current-current" or "tree-level" operators, while we call $\mathcal{O}_{i_{3}}$ "one-loop" operators. $^{4}$

Although our basis follows a classification inspired by renormalizable weakly-coupled theories, it can also be useful when dealing with strongly-coupled BSM models. For example, if the Higgs or SM fermions arise as composite mesonic states of a strongly-interacting gauge theory with no small parameter, our basis can still give the right parametrization by taking $g_{*} \sim 4 \pi$. Also, strongly-coupled models that admit a weakly-coupled holographic description generate $d=6$ operators that follow the above classification. In this case we have $g_{*} \sim 4 \pi / \sqrt{N}$ where $N$ plays the role of the number of colors of the strong sector.

Let us start defining our basis by considering first operators made of SM bosons only [4]. In the first class of operators, $\mathcal{O}_{i_{1}}$, we have

$$
\mathcal{O}_{H}=\frac{1}{2}\left(\partial^{\mu}|H|^{2}\right)^{2} \quad, \quad \mathcal{O}_{T}=\frac{1}{2}\left(H^{\dagger} \stackrel{\leftrightarrow}{D}_{\mu} H\right)^{2} \quad, \quad \mathcal{O}_{r}=|H|^{2}\left|D_{\mu} H\right|^{2} \quad, \quad \mathcal{O}_{6}=\lambda|H|^{6} .
$$

Here we have defined $H^{\dagger} \stackrel{\leftrightarrow}{D_{\mu}} H \equiv H^{\dagger} D_{\mu} H-\left(D_{\mu} H\right)^{\dagger} H$, with $D_{\mu} H=\partial_{\mu} H-i g \sigma^{a} W_{\mu}^{a} H / 2-$ $i g^{\prime} B_{\mu} H / 2\left(H\right.$ is taken to have hypercharge $\left.Y_{H}=1 / 2\right)$. For $\mathcal{O}_{6}$, which involves six Higgs fields, an extra factor $g_{*}^{2}$ could be present. Nevertheless, we have substituted this by $\lambda$, the Higgs self-coupling defined as $V=-m^{2}|H|^{2}+\lambda|H|^{4}$. This is motivated by the fact that the lightness of the Higgs suggests that there is a symmetry protecting the Higgs self-coupling to be of order $\lambda \sim m_{h}^{2} /\left(2 v^{2}\right) \sim 0.13$. Examples are supersymmetry or global symmetries as in composite Higgs models.

In the second class of operators, $\mathcal{O}_{i_{2}}$, we have

$$
\begin{aligned}
& \mathcal{O}_{W}=\frac{i g}{2}\left(H^{\dagger} \sigma^{a} \stackrel{\leftrightarrow}{D^{\mu}} H\right) D^{\nu} W_{\mu \nu}^{a} \quad, \quad \mathcal{O}_{B}=\frac{i g^{\prime}}{2}\left(H^{\dagger} \stackrel{\leftrightarrow}{D^{\mu}} H\right) \partial^{\nu} B_{\mu \nu} \\
& \mathcal{O}_{2 W}=-\frac{1}{2}\left(D^{\mu} W_{\mu \nu}^{a}\right)^{2}, \quad \mathcal{O}_{2 B}=-\frac{1}{2}\left(\partial^{\mu} B_{\mu \nu}\right)^{2}, \quad \mathcal{O}_{2 G}=-\frac{1}{2}\left(D^{\mu} G_{\mu \nu}^{A}\right)^{2} .
\end{aligned}
$$

Since the last three operators involve two field strengths, we expect $c_{2 W} \sim g^{2} / g_{*}^{2}, c_{2 B} \sim g^{\prime 2} / g_{*}^{2}$, and $c_{2 G} \sim g_{s}^{2} / g_{*}^{2}$.

\footnotetext{
${ }^{4}$ For a classification of operators similar in spirit to ours, see [10].
} 
In the third class of operators, $\mathcal{O}_{i_{3}}$, we have the CP-even operators

$$
\begin{aligned}
& \mathcal{O}_{B B}=g^{2}|H|^{2} B_{\mu \nu} B^{\mu \nu}, \quad \mathcal{O}_{G G}=g_{s}^{2}|H|^{2} G_{\mu \nu}^{A} G^{A \mu \nu}, \\
& \mathcal{O}_{H W}=i g\left(D^{\mu} H\right)^{\dagger} \sigma^{a}\left(D^{\nu} H\right) W_{\mu \nu}^{a}, \quad \mathcal{O}_{H B}=i g^{\prime}\left(D^{\mu} H\right)^{\dagger}\left(D^{\nu} H\right) B_{\mu \nu}, \\
& \mathcal{O}_{3 W}=\frac{1}{3 !} g \epsilon_{a b c} W_{\mu}^{a \nu} W_{\nu \rho}^{b} W^{c \rho \mu}, \quad \mathcal{O}_{3 G}=\frac{1}{3 !} g_{s} f_{A B C} G_{\mu}^{A \nu} G_{\nu \rho}^{B} G^{C \rho \mu},
\end{aligned}
$$

and the CP-odd operators

$$
\begin{aligned}
& \mathcal{O}_{B \widetilde{B}}=g^{\prime 2}|H|^{2} B_{\mu \nu} \widetilde{B}^{\mu \nu}, \quad \mathcal{O}_{G \widetilde{G}}=g_{s}^{2}|H|^{2} G_{\mu \nu}^{A} \widetilde{G}^{A \mu \nu}, \\
& \mathcal{O}_{H \widetilde{W}}=i g\left(D^{\mu} H\right)^{\dagger} \sigma^{a}\left(D^{\nu} H\right) \widetilde{W}_{\mu \nu}^{a}, \quad \mathcal{O}_{H \widetilde{B}}=i g^{\prime}\left(D^{\mu} H\right)^{\dagger}\left(D^{\nu} H\right) \widetilde{B}_{\mu \nu}, \\
& \mathcal{O}_{3 \widetilde{W}}=\frac{1}{3 !} g \epsilon_{a b c} \widetilde{W}_{\mu}^{a \nu} W_{\nu \rho}^{b} W^{c \rho \mu} \quad, \quad \mathcal{O}_{3 \widetilde{G}}=\frac{1}{3 !} g_{s} f_{A B C} \widetilde{G}_{\mu}^{A \nu} G_{\nu \rho}^{B} G^{C \rho \mu},
\end{aligned}
$$

where $\widetilde{F}^{\mu \nu}=\epsilon^{\mu \nu \rho \sigma} F_{\rho \sigma} / 2$. There are two more CP-even operators involving two Higgs fields and gauge bosons, $\mathcal{O}_{W B}=g^{\prime} g H^{\dagger} \sigma^{a} H W_{\mu \nu}^{a} B^{\mu \nu}$ and $\mathcal{O}_{W W}=g^{2}|H|^{2} W_{\mu \nu}^{a} W^{\mu \nu a}$ (and the equivalent CP-odd ones), but these can be eliminated using the identities ${ }^{5}$

$$
\begin{aligned}
& \mathcal{O}_{B}=\mathcal{O}_{H B}+\frac{1}{4} \mathcal{O}_{B B}+\frac{1}{4} \mathcal{O}_{W B} \\
& \mathcal{O}_{W}=\mathcal{O}_{H W}+\frac{1}{4} \mathcal{O}_{W W}+\frac{1}{4} \mathcal{O}_{W B} .
\end{aligned}
$$

The operators $\mathcal{O}_{3 W}$ and $\mathcal{O}_{3 G}$ (and the corresponding CP-odd ones) have three field-strengths and then their corresponding coefficients should scale as $c_{3 W} \sim g^{2} / g_{*}^{2}$ and $c_{3 G} \sim g_{s}^{2} / g_{*}^{2}$ respectively.

Let us now examine $d=6$ operators involving SM fermions, considering a single family to begin with. Operators of the first class involving the up-type quark are

$$
\begin{aligned}
\mathcal{O}_{y_{u}} & =y_{u}|H|^{2} \bar{Q}_{L} \widetilde{H} u_{R} \\
\mathcal{O}_{R}^{u} & =\left(i H^{\dagger} \stackrel{\leftrightarrow}{D}_{\mu} H\right)\left(\bar{u}_{R} \gamma^{\mu} u_{R}\right) \\
\mathcal{O}_{L}^{q} & =\left(i H^{\dagger} \stackrel{\leftrightarrow}{D_{\mu} H}\right)\left(\bar{Q}_{L} \gamma^{\mu} Q_{L}\right) \\
\mathcal{O}_{L}^{(3) q} & =\left(i H^{\dagger} \sigma^{a} \stackrel{\leftrightarrow}{D_{\mu}} H\right)\left(\bar{Q}_{L} \gamma^{\mu} \sigma^{a} Q_{L}\right)
\end{aligned}
$$

where $\widetilde{H}=i \sigma_{2} H^{*}$, and in operators $\propto \bar{Q}_{L} u_{R}$ we include a Yukawa coupling $y_{u}\left(m_{u}=y_{u} v / \sqrt{2}\right)$ as an order parameter of the chirality-flip. We also understand, here and in the following, that when needed the Hermitian conjugate of a given operator is included in the analysis. In the first class we have, in addition, the four-fermion operators:

$$
\begin{aligned}
\mathcal{O}_{L L}^{q}=\left(\bar{Q}_{L} \gamma^{\mu} Q_{L}\right)\left(\bar{Q}_{L} \gamma^{\mu} Q_{L}\right), & \mathcal{O}_{L L}^{(8) q}=\left(\bar{Q}_{L} \gamma^{\mu} T^{A} Q_{L}\right)\left(\bar{Q}_{L} \gamma^{\mu} T^{A} Q_{L}\right) \\
\mathcal{O}_{L R}^{u}=\left(\bar{Q}_{L} \gamma^{\mu} Q_{L}\right)\left(\bar{u}_{R} \gamma^{\mu} u_{R}\right), & \mathcal{O}_{L R}^{(8) u}=\left(\bar{Q}_{L} \gamma^{\mu} T^{A} Q_{L}\right)\left(\bar{u}_{R} \gamma^{\mu} T^{A} u_{R}\right) \\
\mathcal{O}_{R R}^{u}=\left(\bar{u}_{R} \gamma^{\mu} u_{R}\right)\left(\bar{u}_{R} \gamma^{\mu} u_{R}\right), &
\end{aligned}
$$

\footnotetext{
${ }^{5}$ For CP-odd operators the identities are $4 \mathcal{O}_{H \widetilde{B}}+\mathcal{O}_{B \widetilde{B}}+\mathcal{O}_{W \widetilde{B}}=0$ and $4 \mathcal{O}_{H \widetilde{W}}+\mathcal{O}_{W \widetilde{W}}+\mathcal{O}_{W \widetilde{B}}=0$.
} 
where $T^{A}$ are the $S U(3)_{c}$ generators. Other four-fermion operators are linear combinations of the ones appearing in Eq. (15); see for example [9,11]. Finally, the one-loop (dipole) operators involving the up-type quark are

$$
\begin{aligned}
\mathcal{O}_{D B}^{u} & =y_{u} \bar{Q}_{L} \sigma^{\mu \nu} u_{R} \widetilde{H} g^{\prime} B_{\mu \nu}, \\
\mathcal{O}_{D W}^{u} & =y_{u} \bar{Q}_{L} \sigma^{\mu \nu} u_{R} \sigma^{a} \widetilde{H} g W_{\mu \nu}^{a}, \\
\mathcal{O}_{D G}^{u} & =y_{u} \bar{Q}_{L} \sigma^{\mu \nu} T^{A} u_{R} \widetilde{H} g_{s} G_{\mu \nu}^{A} .
\end{aligned}
$$

Similar operators to those given above can be written for the down-type quarks and leptons. For one family of fermions these are given in Table 2. Among them, there is a new type of operators, involving two different types of fermions, which, as we will see, can have an important impact on Higgs physics at the one-loop level. These are

$$
\mathcal{O}_{R}^{u d}=y_{u}^{\dagger} y_{d}\left(i \widetilde{H}^{\dagger} \stackrel{\leftrightarrow}{D_{\mu}} H\right)\left(\bar{u}_{R} \gamma^{\mu} d_{R}\right)
$$

and

$$
\begin{aligned}
\mathcal{O}_{y_{u} y_{d}}=y_{u} y_{d}\left(\bar{Q}_{L}^{r} u_{R}\right) \epsilon_{r s}\left(\bar{Q}_{L}^{s} d_{R}\right), & \mathcal{O}_{y_{u} y_{d}}^{(8)}=y_{u} y_{d}\left(\bar{Q}_{L}^{r} T^{A} u_{R}\right) \epsilon_{r s}\left(\bar{Q}_{L}^{s} T^{A} d_{R}\right) \\
\mathcal{O}_{y_{u} y_{e}}=y_{u} y_{e}\left(\bar{Q}_{L}^{r} u_{R}\right) \epsilon_{r s}\left(\bar{L}_{L}^{s} e_{R}\right), & \mathcal{O}_{y_{u} y_{e}}^{\prime}=y_{u} y_{e}\left(\bar{Q}_{L}^{r \alpha} e_{R}\right) \epsilon_{r s}\left(\bar{L}_{L}^{s} u_{R}^{\alpha}\right) \\
\mathcal{O}_{y_{e} y_{d}}=y_{e} y_{d}^{\dagger}\left(\bar{L}_{L} e_{R}\right)\left(\bar{d}_{R} Q_{L}\right), &
\end{aligned}
$$

where $\epsilon=i \sigma_{2}$ and $\alpha$ labels color (only shown when contracted outside parentheses). These operators are in principle of the first type. Nevertheless in the four-fermion operators of Eq. (18) we have incorporated a product of Yukawa couplings since they involve two chiralityflips, while in Eq. (17) we have also included Yukawa couplings as it is the case in theories with a flavour symmetry, as discussed below. These operators are then only suppressed by $1 / \Lambda^{2}$ as second-class operators.

There is some redundancy in the operators given above, as it is clear that some of them can be eliminated by field redefinitions (see Appendix A) or using the equations of motion (EoM). For example, the operator $\mathcal{O}_{r}$ can be eliminated by field redefinitions:

$$
c_{r} \mathcal{O}_{r} \leftrightarrow c_{r}\left[\frac{1}{2}\left(\mathcal{O}_{y_{u}}+\mathcal{O}_{y_{d}}+\mathcal{O}_{y_{e}}+\text { h.c. }\right)-\mathcal{O}_{H}+2 \mathcal{O}_{6}\right] .
$$

Also, we could eliminate all 5 operators of Eq. (5) by using the EoM for the gauge fields:

$$
\begin{aligned}
D^{\nu} W_{\mu \nu}^{a} & =i g H^{\dagger} \frac{\sigma^{a}}{2} \stackrel{\leftrightarrow}{D}_{\mu} H+g \sum_{f} \bar{f}_{L} \frac{\sigma^{a}}{2} \gamma_{\mu} f_{L} \\
\partial^{\nu} B_{\mu \nu} & =i g^{\prime} Y_{H} H^{\dagger} \stackrel{\leftrightarrow}{D}_{\mu} H+g^{\prime} \sum_{f}\left[Y_{L}^{f} \bar{f}_{L} \gamma_{\mu} f_{L}+Y_{R}^{f} \bar{f}_{R} \gamma_{\mu} f_{R}\right] \\
D^{\nu} G_{\mu \nu}^{A} & =g_{s} \sum_{q} \bar{q} T^{A} \gamma_{\mu} q
\end{aligned}
$$




\begin{tabular}{|c|}
\hline $\begin{array}{c}\mathcal{O}_{H}=\frac{1}{2}\left(\partial^{\mu}|H|^{2}\right)^{2} \\
\mathcal{O}_{T}=\frac{1}{2}\left(H^{\dagger} \stackrel{\leftrightarrow}{D}_{\mu} H\right)^{2} \\
\mathcal{O}_{6}=\lambda|H|^{6}\end{array}$ \\
\hline $\begin{array}{c}\mathcal{O}_{W}=\frac{i g}{2}\left(H^{\dagger} \sigma^{a} \stackrel{\leftrightarrow}{D^{\mu}} H\right) D^{\nu} W_{\mu \nu}^{a} \\
\mathcal{O}_{B}=\frac{i g^{\prime}}{2}\left(H^{\dagger} \stackrel{\leftrightarrow}{D^{\mu}} H\right) \partial^{\nu} B_{\mu \nu} \\
\mathcal{O}_{2 W}=-\frac{1}{2}\left(\bar{D}^{\mu} W_{\mu \nu}^{\bar{a}}\right)^{2}- \\
\mathcal{O}_{2 B}=-\frac{1}{2}\left(\partial^{\mu} B_{\mu \nu}\right)^{2} \\
\mathcal{O}_{2 G}=-\frac{1}{2}\left(D^{\mu} G_{\mu \nu}^{A}\right)^{2}\end{array}$ \\
\hline 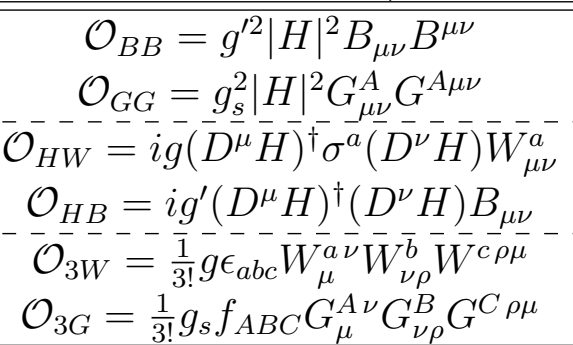 \\
\hline
\end{tabular}

Table 1: 14 CP-even operators made of SM bosons. The operators are grouped in 3 different boxes corresponding to the 3 classes of operators defined in Eq. (2). Dashed lines separate operators of different structure within a given class. There are, in addition, the 6 CP-odd operators given in Eqs. (9)-(11).

where $Y_{L, R}^{f}$ are the fermion hypercharges and $Y_{H}$ the Higgs hypercharge. In particular, we could trade $\mathcal{O}_{B}$ and $\mathcal{O}_{W}$ with other operators:

$$
\begin{aligned}
c_{B} \mathcal{O}_{B} & \leftrightarrow c_{B} \frac{g^{\prime 2}}{g_{*}^{2}}\left[-\frac{1}{2} \mathcal{O}_{T}+\frac{1}{2} \sum_{f}\left(Y_{L}^{f} \mathcal{O}_{L}^{f}+Y_{R}^{f} \mathcal{O}_{R}^{f}\right)\right], \\
c_{W} \mathcal{O}_{W} & \leftrightarrow c_{W} \frac{g^{2}}{g_{*}^{2}}\left[-\frac{3}{2} \mathcal{O}_{H}+2 \mathcal{O}_{6}+\frac{1}{2}\left(\mathcal{O}_{y_{u}}+\mathcal{O}_{y_{d}}+\mathcal{O}_{y_{e}}+\text { h.c. }\right)+\frac{1}{4} \sum_{f} \mathcal{O}_{L}^{(3) f}\right],
\end{aligned}
$$

where, in the last expression, we have eliminated $\mathcal{O}_{r}$ using Eq. (19).

For one family of fermions the set of operators that we use is collected in Tables 1 and 2 . We keep all operators of Eqs. (4)-(11), since they are the relevant ones for a well-motivated class of BSM scenarios such as universal theories, with the exception of $\mathcal{O}_{r}$, that we eliminate of our basis using Eq. (19). In Tables 1 and 2 there are 58 operators; adding the 6 bosonic CPodd ones in Eqs. (9)-(11) leads to a total of 64 operators. We still have 5 redundant operators that once eliminated leave a total of 59 independent operators, in agreement with [9]. We leave free the choice of which 5 operators to eliminate: e.g., the operators of Eq. (5) could be eliminated by using Eq. (20) or, alternatively, we could trade 5 operators that contain fermions by the operators in Eq. (5). We will use later this freedom in different ways depending on the physics process studied. Other redundant operators are discussed in Appendix A. 


\begin{tabular}{|c|c|c|c|}
\hline 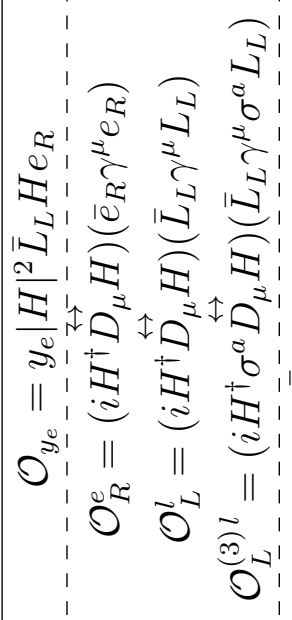 & : & & 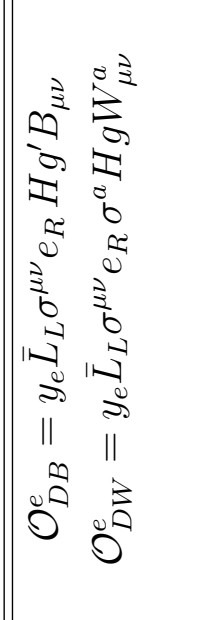 \\
\hline 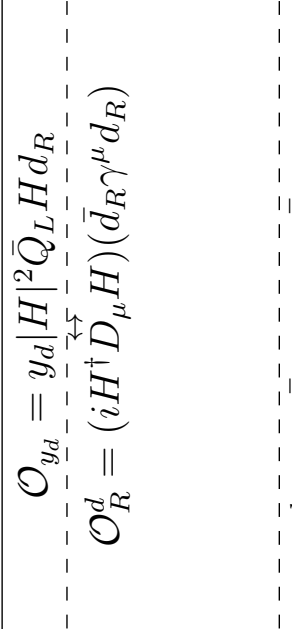 & 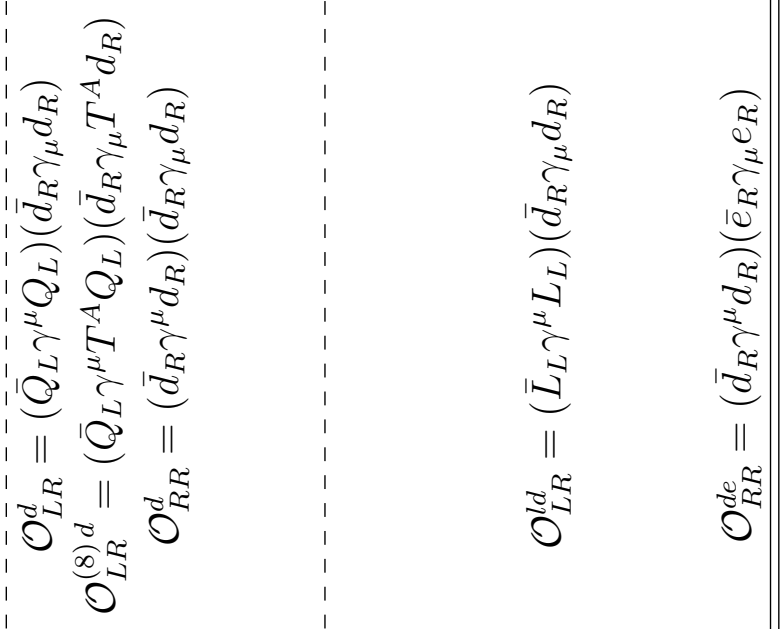 & & 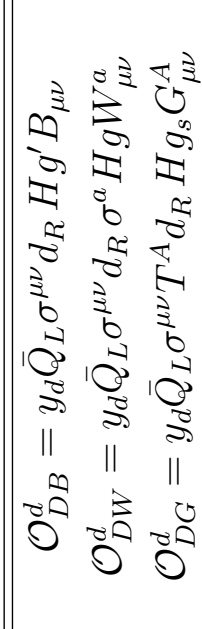 \\
\hline 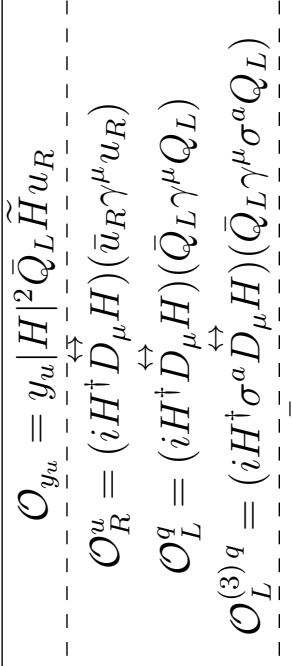 & 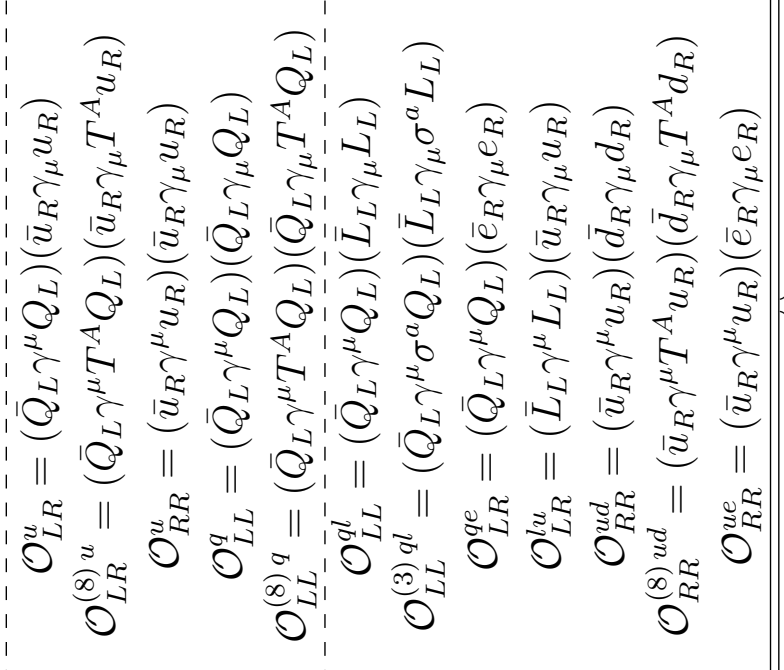 & 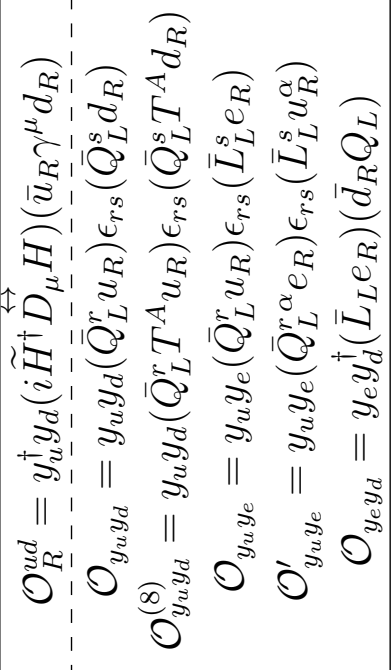 & 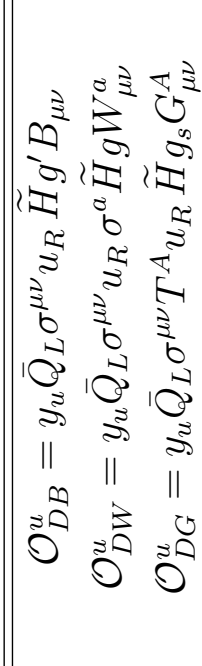 \\
\hline
\end{tabular}

Table 2: 44 operators made of one-family of SM fermions. In the first column there are operators made of the up-type quark and other fermions; in the second column there are operators made only of the down-type quark and leptons; the third column lists operators made only of leptons. The operators are grouped in 3 different boxes corresponding to the 3 classes of operators defined in Eq. (2). Dashed lines separate operators of different structure within a given class. 
Extending the basis to 3 families increases considerably the number of operators. We can reduce it by imposing flavor symmetries, which are also needed to avoid tight constraints on flavor-violating processes. For example, we can require the BSM sector to be invariant under the flavor symmetry $U(3)_{Q_{L}} \otimes U(3)_{d_{R}} \otimes U(3)_{u_{R}} \otimes U(3)_{L_{L}} \otimes U(3)_{e_{R}}$, under which the corresponding 3 families transform as triplets, and the Yukawas become $3 \times 3$ matrices transforming as $y_{d} \in(\mathbf{3}, \overline{\mathbf{3}}, \mathbf{0}, \mathbf{0}, \mathbf{0}), y_{u} \in(\mathbf{3}, \mathbf{0}, \overline{\mathbf{3}}, \mathbf{0}, \mathbf{0})$ and $y_{e} \in(\mathbf{0}, \mathbf{0}, \mathbf{0}, \mathbf{3}, \overline{\mathbf{3}})$ under the non-Abelian part of the flavor group. One can also assume that the Yukawas are the only source of CP violation. This assumption goes under the name of Minimal Flavor Violation (MFV) [12]. In this case the list of operators given in Table 2 can be easily generalized to include 3 families. For example, for operators involving two fermions, we have

$$
\begin{aligned}
\left(\bar{L}_{L} \gamma^{\mu} L_{L}\right) & \rightarrow\left[\delta_{i j}+O\left(y_{e} y_{e}^{\dagger} / g_{*}^{2}\right)\right]\left(\bar{L}_{L}^{i} \gamma^{\mu} L_{L}^{j}\right) \\
y_{e} \bar{L}_{L} e_{R} & \rightarrow y_{e}^{i j}\left[1+O\left(y_{e}^{\dagger} y_{e} / g_{*}^{2}\right)\right] \bar{L}_{L}^{i} e_{R}^{j},
\end{aligned}
$$

$(i, j$ are family indices) and similarly for other fermion species. For 4-fermion operators, we have several possibilities to form singlets under the flavor group. For the leptons we find four independent operators:

$$
\begin{aligned}
\mathcal{O}_{L L}^{l} & =\left(\bar{L}_{L}^{i} \gamma^{\mu} L_{L}^{i}\right)\left(\bar{L}_{L}^{j} \gamma_{\mu} L_{L}^{j}\right) \\
\mathcal{O}_{L L}^{(3) l} & =\left(\bar{L}_{L}^{i} \gamma^{\mu} \sigma^{a} L_{L}^{i}\right)\left(\bar{L}_{L}^{j} \gamma_{\mu} \sigma^{a} L_{L}^{j}\right) \\
\mathcal{O}_{L R}^{e} & =\left(\bar{L}_{L}^{i} \gamma^{\mu} L_{L}^{i}\right)\left(\bar{e}_{R}^{j} \gamma_{\mu} e_{R}^{j}\right) \\
\mathcal{O}_{R R}^{e} & =\left(\bar{e}_{R}^{i} \gamma^{\mu} e_{R}^{i}\right)\left(\bar{e}_{R}^{j} \gamma_{\mu} e_{R}^{j}\right)
\end{aligned}
$$

where we are neglecting terms of $O\left(y_{e}^{2} / g_{*}^{2}\right)$, while the independent set of 4-quark operators can be found in the Appendix of Ref. [13]. The MFV assumption that the Yukawas are the only source of CP violation implies that the Wilson coefficients are real. For the top quark, having a Yukawa coupling of order one, departures from flavor-universality could be important.

It is useful, in order to understand what operators mix under the RGE, to derive the transformation of the coefficients (or equivalently, of the operators) under the global custodial $S U(2)_{L} \otimes S U(2)_{R}$ symmetry and the parity $P_{L R}$ that interchanges $L \leftrightarrow R$. A detailed analysis is given in Appendix C. In Table 3 we present the quantum numbers of the coefficients of the tree-level operators involving the Higgs.

\section{$3 \quad$ Higgs physics}

Let us now describe the effects of the $d=6$ operators on Higgs physics. We will only present the modifications of the Higgs couplings important for single Higgs production and decay, working under the assumption of MFV, allowing however for CP-violating bosonic operators. We split the relevant part of the Lagrangian in two parts,

$$
\mathcal{L}_{h}=\mathcal{L}_{h}^{(0)}+\mathcal{L}_{h}^{(1)}
$$




\begin{tabular}{|c|c|c|}
\hline Spurion & $S U(2)_{L} \otimes S U(2)_{R}$ & $P_{L R}$ \\
\hline \hline$y_{f}$ & $\mathbf{2}_{R}$ & \\
\hline$g^{\prime}$ & $\mathbf{3}_{R}+\mathbf{1}$ & \\
\hline \hline$c_{T}$ & $\left(\mathbf{3}_{R} \otimes \mathbf{3}_{R}\right)_{s}$ & \\
\hline$c_{H}, c_{6}$ & $\mathbf{1}$ & + \\
\hline$c_{B}+c_{W}$ & $\mathbf{1}$ & + \\
\hline$c_{B}-c_{W}$ & $\mathbf{1}$ & - \\
\hline$c_{y_{f}}$ & $\mathbf{1}$ & \\
\hline$c_{R}^{f}$ & $\mathbf{3}_{R}$ & \\
\hline$c_{L}^{f}$ & $\mathbf{3}_{R}$ & \\
\hline$c_{L}^{(3) f}$ & $\mathbf{1}$ & \\
\hline$c_{R}^{u d}$ & $\mathbf{1}$ & \\
\hline
\end{tabular}

Table 3: Quantum numbers under the custodial $S U(2)_{L} \otimes S U(2)_{R}$ and left-right parity $P_{L R}$ of the SM couplings and coefficients of the tree-level operators involving Higgs fields. We only show the $P_{L R}$-parities of the coefficients with a well-defined transformation, see Apendix $C$.

In $\mathcal{L}_{h}^{(0)}$ we keep the SM couplings and the effects of the current-current operators of Tables 1 and 2, while $\mathcal{L}_{h}^{(1)}$ has the effects of the loop operators. We can remove the momentum dependence from the Higgs couplings in $\mathcal{L}_{h}^{(0)}$ by using the EoM, so that we end up with Higgs couplings at zero momentum. After doing that, we have, in the canonical basis for the Higgs field $h$,

$$
\begin{aligned}
\mathcal{L}_{h}^{(0)}= & g_{h f f} h\left(\bar{f}_{L} f_{R}+\text { h.c. }\right)+g_{h V V} h V^{\mu} V_{\mu}+g_{h Z f_{L} f_{L}} h Z_{\mu} \bar{f}_{L} \gamma^{\mu} f_{L} \\
& +g_{h Z f_{R} f_{R}} h Z_{\mu} \bar{f}_{R} \gamma^{\mu} f_{R}+g_{h W f_{L} f_{L}^{\prime}} h W_{\mu} \bar{f}_{L} \gamma^{\mu} f_{L}^{\prime},
\end{aligned}
$$


where a sum over fermions is understood and $V=W, Z$. The couplings read ${ }^{6}$

$$
\begin{aligned}
g_{h f f} & =g_{h f f}^{\mathrm{SM}}\left[1-\left(\frac{c_{H}}{2}+c_{y_{f}}\right) \xi+\frac{\delta G_{F}}{2 G_{F}}\right] \\
g_{h W W} & =g_{h W W}^{\mathrm{SM}}\left[1-\left(c_{H}-\frac{g^{2}}{g_{*}^{2}} c_{W}\right) \frac{\xi}{2}+\frac{\delta G_{F}}{2 G_{F}}+2 \frac{\delta M_{W}}{M_{W}}\right], \\
g_{h Z Z} & =g_{h Z Z}^{\mathrm{SM}}\left[1-\left(c_{H}-\frac{g^{2}}{g_{*}^{2}} c_{Z}\right) \frac{\xi}{2}-\widehat{T}+\frac{\delta G_{F}}{2 G_{F}}\right] \\
g_{h W f_{L} f_{L}^{\prime}} & =\frac{1}{2 \sqrt{2} v} \frac{g^{3}}{g_{*}^{2}} c_{W} \xi+\frac{2}{v} \delta g_{W}^{f_{L}}, \\
g_{h Z f_{L} f_{L}} & =\frac{1}{2 v \cos \theta_{W}} \frac{g^{3}}{g_{*}^{2}}\left(T_{L}^{3} c_{Z}-Q_{f} c_{B} \tan ^{2} \theta_{W}\right) \xi+\frac{2}{v} \delta g_{Z}^{f_{L}}, \\
g_{h Z f_{R} f_{R}} & =-\frac{\tan ^{2} \theta_{W}}{2 v \cos \theta_{W}} \frac{g^{3}}{g_{*}^{2}} Q_{f} c_{B} \xi+\frac{2}{v} \delta g_{Z}^{f_{R}} .
\end{aligned}
$$

Here the SM couplings must be expressed as a function of the input parameters $\alpha=e^{2} /(4 \pi)$, the Fermi constant $G_{F}$ and the physical $m_{h}, M_{Z}$ and fermion masses. In these equations, $\theta_{W}$ is the weak mixing angle, $T_{L}^{3}= \pm 1 / 2$ stands for the weak isospin values of up and down components of $S U(2)_{L}$ fermion doublets, $Q_{f}$ is the fermion electric charge. We have defined

$$
\xi \equiv \frac{g_{*}^{2} v^{2}}{\Lambda^{2}}
$$

with $v \simeq 246 \mathrm{GeV}$, and

$$
c_{Z}=c_{W}+\tan ^{2} \theta_{W} c_{B}
$$

In the couplings of Eq. (26), we have introduced

$$
\begin{aligned}
\frac{\delta G_{F}}{G_{F}} & =2\left[c_{L L}^{(3) l}-c_{L}^{(3) l}\right] \xi, \\
\frac{\delta M_{W}}{M_{W}} & =\frac{1}{2\left(1-2 \sin ^{2} \theta_{W}\right)}\left[\cos ^{2} \theta_{W} \widehat{T}-2 \sin ^{2} \theta_{W} \widehat{S}+\sin ^{2} \theta_{W} \frac{\delta G_{F}}{G_{F}}\right],
\end{aligned}
$$

and

$$
\begin{aligned}
\delta g_{W}^{f_{L}} & =\frac{g}{\sqrt{2}} c_{L}^{(3) f} \xi \\
\delta g_{Z}^{f_{L}} & =\frac{g}{2 \cos \theta_{W}}\left(2 T_{L}^{3} c_{L}^{(3) f}-c_{L}^{f}\right) \xi \\
\delta g_{Z}^{f_{R}} & =-\frac{g}{2 \cos \theta_{W}} c_{R}^{f} \xi .
\end{aligned}
$$

Finally, we have made use of the precision electroweak parameters $[14,15]$

$$
\widehat{S}=\left(c_{W}+c_{B}\right) \frac{M_{W}^{2}}{\Lambda^{2}}, \quad \widehat{T}=c_{T} \xi
$$

\footnotetext{
${ }^{6}$ A coupling of $W_{\mu}^{ \pm}$to the right-handed current $\bar{f}_{R} \gamma^{\mu} f_{R}^{\prime}$ is generated from the operator $\mathcal{O}_{R}^{u d}$ in Eq. (17), but we do not include it as it is expected to be suppressed by two Yukawa couplings (due to the MFV assumption) and hence to be small.
} 
As we have stressed in the previous Section, not all the operators appearing in the Higgs couplings of Eq. (26) are independent. Once one has decided which are the redundant operators which are not in the basis, one should simply put equal to zero the corresponding operator coefficients.

The second term in the Lagrangian (24) necessarily contains field derivatives. It reads

$$
\begin{aligned}
\mathcal{L}_{h}^{(1)} & =g_{\partial h W W}\left(W^{+\mu} W_{\mu \nu}^{-} \partial^{\nu} h+\text { h.c. }\right)+g_{\partial h Z Z} Z^{\mu} Z_{\mu \nu} \partial^{\nu} h+g_{h Z Z}^{\prime} h Z^{\mu \nu} Z_{\mu \nu} \\
& +g_{h A A} h A^{\mu \nu} A_{\mu \nu}+g_{\partial h A Z} Z^{\mu} A_{\mu \nu} \partial^{\nu} h+g_{h A Z} h A^{\mu \nu} Z_{\mu \nu}+g_{h G G} h G^{A \mu \nu} G_{\mu \nu}^{A},
\end{aligned}
$$

where we have defined $V_{\mu \nu}=\partial_{\mu} V_{\nu}-\partial_{\nu} V_{\mu}$, for $V=W^{ \pm}, Z, A$. The couplings are given by

$$
\begin{aligned}
g_{\partial h W W} & =-\frac{g^{2} v}{2 \Lambda^{2}} \kappa_{H W}, \\
g_{\partial h Z Z} & =-\frac{g^{2} v}{2 \Lambda^{2}}\left(\kappa_{H W}+\kappa_{H B} \tan ^{2} \theta_{W}\right), \\
g_{h A A} & =\frac{e^{2} v}{\Lambda^{2}} \kappa_{B B}=\frac{g_{h Z Z}^{\prime}}{\tan ^{2} \theta_{W}}=-\frac{g_{h A Z}}{2 \tan \theta_{W}}, \\
g_{\partial h A Z} & =-\frac{g^{2} v}{2 \Lambda^{2}} \tan \theta_{W}\left(\kappa_{H W}-\kappa_{H B}\right), \\
g_{h G G} & =\frac{g_{s}^{2} v}{\Lambda^{2}} \kappa_{G G} .
\end{aligned}
$$

The contributions from the CP-violating bosonic operators can be easily obtained from Eq. (33) by replacing one of the field strengths $F_{\mu \nu}$ in the operators by $\widetilde{F}_{\mu \nu}$. Only the contributions from the dipole operators (third box of Table 2) have been neglected since they are assumed to be proportional to Yukawa couplings.

In the list of modified Higgs couplings (26), the tree-level operator $\mathcal{O}_{6}$ does not play any role. The simplest modified coupling containing this operator would be the triple Higgs vertex

$$
\delta \mathcal{L}_{h}^{(0)}=g_{h h h}^{\mathrm{SM}}\left[1-\left(c_{6}+\frac{3 c_{H}}{2}\right) \xi+\frac{\delta G_{F}}{2 G_{F}}\right] h^{3},
$$

where $g_{h h h}^{\mathrm{SM}}$ is the SM value for the $h^{3}$ coupling. Experimental access to this coupling is not yet possible.

From the couplings in Eqs. (25) and (33) it is easy to derive the modifications of the main Higgs partial-widths due to $d=6$ operators [3-5]. ${ }^{7}$ The coefficients $c_{L}^{(3) f}, c_{L}^{f}, c_{R}^{f}$ can also modify the cross-section of $h f \bar{f}$ production, giving contributions that grow with the energy. A particularly interesting case is $p p \rightarrow q t h$ ( $q$ being a light quark) that is dominated by the subprocess $W_{L} b \rightarrow t h$. At large energies this grows with the energy as

$$
\left|\mathcal{A}\left(W_{L} b \rightarrow t h\right)\right|^{2} \simeq\left(\frac{4 g_{*}^{2} c_{L}^{(3) q_{3}}}{\Lambda^{2}}\right)^{2} s(s+t) .
$$

The extraction of new physics through this process has been studied in Ref. [16].

\footnotetext{
${ }^{7}$ For loop-suppressed partial-widths, such as $h \rightarrow \gamma \gamma$, we remind the reader that $d=6$ operators can have an effect either directly or through modifications of the SM couplings that change the SM loop contribution to that particular decay [4].
} 


\section{Experimental constraints on the Wilson coefficients}

As we saw in the previous Section, many $d=6$ operators can directly affect the Higgs couplings. Some of them only affect Higgs physics (at tree-level). Their corresponding coefficients are

$$
\left\{c_{H}, c_{6}, c_{y_{f}}, \kappa_{B B}, \kappa_{G G}, \hat{\kappa}_{W W}, \kappa_{B \widetilde{B}}, \kappa_{G \widetilde{G}}, \hat{\kappa}_{W \widetilde{W}}\right\} .
$$

The reason for this is clear in the case of $c_{H}$ and $c_{6}$ as these operators contain exclusively Higgs fields; and in the case of $c_{y_{f}}, \kappa_{B B}$ and $\kappa_{G G}$ because, when the Higgs is substituted by its vacuum expectation value (VEV), these operators simply lead to an innocuous renormalisation of SM parameters. The coefficient $\hat{\kappa}_{W W}$ corresponds to the direction in parameter space given by ${ }^{8}$

$$
\kappa_{H B}=-\kappa_{H W}=4 \kappa_{B B}=c_{W}=-c_{B} \equiv 4 \hat{\kappa}_{W W},
$$

and the reason why this direction is only constrained by Higgs physics is subtle in our basis. The easiest way to see it is to go from our basis, that contains the subset

$$
\mathcal{B}_{1}=\left\{\mathcal{O}_{W}, \mathcal{O}_{B}, \mathcal{O}_{H W}, \mathcal{O}_{H B}, \mathcal{O}_{B B}\right\}
$$

to the basis containing the subset $\mathcal{B}_{3}$ defined in [7]:

$$
\mathcal{B}_{3}=\left\{\mathcal{O}_{W}, \mathcal{O}_{B}, \mathcal{O}_{W W}, \mathcal{O}_{W B}, \mathcal{O}_{B B}\right\}
$$

One can go from one to another using (13). Now, in the basis containing $\mathcal{O}_{W W}$ it is clear that its coefficient cannot be bounded by any non-Higgs SM processes, for exactly the same reasons as $\kappa_{B B}$. We can now use Eq. (13) to get the expression of $\mathcal{O}_{W W}$ in terms of the operators in $\mathcal{B}_{1}$,

$$
\mathcal{O}_{W W}=4\left(\mathcal{O}_{W}-\mathcal{O}_{B}\right)-4\left(\mathcal{O}_{H W}-\mathcal{O}_{H B}\right)+\mathcal{O}_{B B}
$$

which leads to the direction given in Eq. (38). Similarly, for the CP-odd operators, $\hat{\kappa}_{W \widetilde{W}}$ corresponds to the direction:

$$
\kappa_{H \widetilde{B}}=-\kappa_{H \widetilde{W}}=4 \kappa_{B \widetilde{B}} \equiv 4 \hat{\kappa}_{W \widetilde{W}} .
$$

Although the coefficients $c_{H}, c_{6}$ and $c_{y_{f}}$ have no severe constraints from Higgs physics yet [18], the coefficients $\kappa_{B B}$ and the difference $\kappa_{H W}-\kappa_{H B}$ are subject to strong constraints from $h \rightarrow \gamma \gamma$ and $h \rightarrow Z \gamma$ respectively (as these decays are one-loop suppressed in the SM). These give at $95 \% \mathrm{CL}[18]$

$$
-0.0013 \lesssim \frac{M_{W}^{2}}{\Lambda^{2}} \kappa_{B B} \lesssim 0.0018, \quad-0.016 \lesssim \frac{M_{W}^{2}}{\Lambda^{2}}\left(\kappa_{H W}-\kappa_{H B}\right) \lesssim 0.009 .
$$

\footnotetext{
${ }^{8}$ In Ref. [17] these were called blind directions, combinations of operators which a certain group of experiments cannot bound. In the case of $\hat{\kappa}_{W W}$ that group is non-Higgs experiments.
} 
Notice that $\kappa_{H W}-\kappa_{H B}$ is odd under $P_{L R}$ [Eq. (C.4)] and could be suppressed with respect to the sum $\kappa_{H W}+\kappa_{H B}$ if the BSM sector respects this parity. Similarly, the coefficient $\kappa_{G G}$ enters in the production $G G \rightarrow h$ and gets the bound [18]:

$$
\frac{M_{W}^{2}}{\Lambda^{2}}\left|\kappa_{G G}\right| \lesssim 0.004
$$

The coefficients of the CP-odd operators enter quadratically in $\Gamma(h \rightarrow \gamma \gamma)$ and $\Gamma(h \rightarrow Z \gamma)$, and therefore their effects are suppressed with respect to CP-even ones.

Apart from the "Higgs-only" coefficients of Eq. (37), the rest of the coefficients of $d=6$ operators that enter in the Lagrangian of Eq. (25) and Eq. (33), relevant for single Higgs physics, can in principle be constrained by (non-Higgs) SM processes. In the following we present the main experimental constraints on these Wilson coefficients. We also discuss limits on other Wilson coefficients that, although do not affect Higgs physics at tree-level, could do it at the one-loop level. The details of this study with a full dedicated quantitative analysis will be presented in [3]. In what follows we assume MFV (unless explicitly stated) and CPinvariance.

\subsection{Universal theories}

We start considering universal theories, leaving the generalization for later. The new physics effects of these theories are captured by the operators listed in Table 1. Deviations in the $W^{ \pm}$and $Z^{0}$ propagators can be parametrized by four quantities, $\widehat{S}, \widehat{T}, W$ and $Y$ [15]. The contributions from $d=6$ operators to $\widehat{S}$ and $\widehat{T}$ have been written in (32); the corresponding equations for $W$ and $Y$ read

$$
W=c_{2 W} \frac{M_{W}^{2}}{\Lambda^{2}}, \quad Y=c_{2 B} \frac{M_{W}^{2}}{\Lambda^{2}} .
$$

LEP1, LEP2 $\left(e^{+} e^{-} \rightarrow l^{+} l^{-}\right)$and Tevatron allow to constrain independently each of these four quantities, all of them at the per-mille level [15]. ${ }^{9}$ We saw in (32) that $\widehat{S}$ depends only on the combination $c_{W}+c_{B}$. The gauge-boson part of the orthogonal combination, $\mathcal{O}_{W}-\mathcal{O}_{B}$, contains at least three gauge bosons

$$
\left.\left(\mathcal{O}_{W}-\mathcal{O}_{B}\right)\right|_{\langle H\rangle}=O\left(V^{3}\right),
$$

and thus it is a blind direction for LEP1 experiments. To constrain this direction, we have to consider the effect of $c_{W, B}$ on triple gauge-boson vertices, which can be cast in the form

$$
\begin{aligned}
\delta \mathcal{L}_{3 V}= & i g \cos \theta_{W}\left[\delta g_{1}^{Z} Z^{\mu}\left(W^{-\nu} W_{\mu \nu}^{+}-W^{+\nu} W_{\mu \nu}^{-}\right)+\delta \kappa_{Z} Z^{\mu \nu} W_{\mu}^{-} W_{\nu}^{+}+\frac{\lambda_{Z}}{M_{W}^{2}} Z^{\mu \nu} W_{\nu}^{-\rho} W_{\rho \mu}^{+}\right] \\
& +i g \sin \theta_{W}\left[\delta \kappa_{\gamma} A^{\mu \nu} W_{\mu}^{-} W_{\nu}^{+}+\frac{\lambda_{\gamma}}{M_{W}^{2}} A^{\mu \nu} W_{\nu}^{-\rho} W_{\rho \mu}^{+}\right]
\end{aligned}
$$

\footnotetext{
${ }^{9} \mathrm{LHC}$ data is also useful to constrain $W, Y$ and $c_{2 G}$, which affect quark cross-sections at high energies [13].
} 
where again we have defined $V_{\mu \nu}=\partial_{\mu} V_{\nu}-\partial_{\nu} V_{\mu}$ for $V=W^{ \pm}, Z, A$. The contributions from $d=6$ operators to these couplings are given by

$$
\begin{aligned}
\delta g_{1}^{Z} & =\frac{M_{Z}^{2}}{\Lambda^{2}}\left(c_{W}+\kappa_{H W}\right) \\
\delta \kappa_{\gamma} & =\frac{M_{W}^{2}}{\Lambda^{2}}\left(\kappa_{H W}+\kappa_{H B}\right) \\
\delta \kappa_{Z} & =\delta g_{1}^{Z}-\tan ^{2} \theta_{W} \delta \kappa_{\gamma}, \\
\lambda_{Z} & =\lambda_{\gamma}=\frac{M_{W}^{2}}{\Lambda^{2}} \kappa_{3 W},
\end{aligned}
$$

where we do not include a contribution from $c_{2 W}$ since it is constrained to be small, as we have seen before. The third relation, as well as the identity $\lambda_{Z}=\lambda_{\gamma}$, are a consequence of limiting the analysis to $d=6$ operators [8]. The best current limits on triple gauge-boson vertices still come from $e^{+} e^{-} \rightarrow W^{+} W^{-}$at LEP2 [19], although LHC results are almost as good and will be better in the near future [20,21]. Leaving aside the contributions from $\kappa_{3 W}$, that we expect to be small in most theories in which the SM gauge bosons are elementary above $\Lambda$, we can use the two-parameter fit from LEP2 [19] which at 95\%CL reads

$$
\begin{aligned}
-0.046 & \leqslant \delta g_{1}^{Z} \leqslant 0.050 \\
-0.11 & \leqslant \delta \kappa_{\gamma} \leqslant 0.084
\end{aligned}
$$

These are a factor $\sim 10$ weaker than the constraints on the coefficients $\widehat{S}, \widehat{T}, W$ and $Y$ from LEP1 (for this reason we can neglect their contributions to $e^{+} e^{-} \rightarrow W^{+} W^{-}$). As expected, the two constraints in Eq. (49) are orthogonal in parameter space to the direction $\hat{\kappa}_{W W}$ of Eq. (38), as can be seen using Eq. (48). For this reason, to obtain independent bounds on the 4 parameters $c_{W}, c_{B}, \kappa_{H B}$ and $\kappa_{H W}$, we need the constraint Eq. (43) combined with Eq. (49) and the bound on $\widehat{S}$. These bounds are at the percent level. In the particular case of $\kappa_{i} \ll c_{i}$, as expected in weakly-coupled theories, we obtain the bound

$$
-0.046 \lesssim \frac{M_{Z}^{2}}{\Lambda^{2}} c_{W} \lesssim 0.050
$$

As we said, LHC tests of triple gauge-boson vertices are becoming comparable to those from LEP2, and it is foreseen that LHC will surpass LEP2 in these type of measurements $[20,21]$. It follows that an important implication of our study is that the LHC will have a direct impact on the improvement of the limits on $c_{W}+\kappa_{H W}, \kappa_{H W}+\kappa_{H B}$ and $\kappa_{3 W}$. We will see in the next Subsection that this conclusion is also valid in non-universal theories.

\subsection{Non-universal theories}

Let us now discuss BSM models without the universal assumption, considering then all operators of the basis. We will follow a different strategy than in the previous Subsection. Let us first look at electroweak leptonic physics for which the experimental constraints are expected 
to be the strongest ones. Since we assume MFV, dipole operators (third box of Table 2) give corrections to SM processes proportional to lepton masses and can then be neglected. We use the redundancy in our set of operators to eliminate, by using Eq. (21), the 5 operators $\mathcal{O}_{2 B, 2 W, 2 G}, \mathcal{O}_{L}^{(3) l}$ and $\mathcal{O}_{L}^{l}$. Taking $\alpha, M_{Z}$ and $G_{F}$ as input parameters, the relevant operators for the leptonic data are the 4 operators $\mathcal{O}_{T}, \mathcal{O}_{W}, \mathcal{O}_{B}, \mathcal{O}_{R}^{e}$ and the four-lepton operators of Eq. (23). LEP1 data and Tevatron afford 4 well-measured experimental quantities: The charged-leptonic width $\Gamma\left(Z \rightarrow l^{+} l^{-}\right)$, the leptonic left-right asymmetry $A_{L R}^{l}$, the $Z$-width into neutrinos $\Gamma(Z \rightarrow \nu \bar{\nu})=\Gamma_{Z}^{\text {total }}-\Gamma_{Z}^{\text {visible }}$ and $M_{W}$. These allow us to place bounds on the 4 quantities $\left\{c_{T}, c_{W}+c_{B}, c_{R}^{e}, \delta G_{F} / G_{F}\right\}$ [where $\delta G_{F} / G_{F}$ is given in Eq. (29)] at almost the same level as for universal theories. We again need the LEP2 constraint of Eq. (49) from $e^{+} e^{-} \rightarrow W^{+} W^{-}$to bound the difference $c_{W}-c_{B}$ [see Eq. (46)]. The only remaining operators are four-lepton interactions but they can also be highly constrained from $e^{+} e^{-} \rightarrow l^{+} l^{-}$ at LEP2.

Having these constraints in mind, we can now move to the quark sector. Higgs-fermion operators, as those in Eq. (14), give contributions to the gauge-boson couplings to quarks that make them depart from the leptonic ones by the amounts $\delta g_{W}^{q_{L}}, \delta g_{Z}^{q_{L}}$ and $\delta g_{Z}^{q_{R}}$ given in (31). Experiments put severe bounds on these deviations. For example, we have limits at the per-mille level on deviations from lepton-quark universality from $\beta$-decays and semileptonic $K$-decays [22]. This implies that the coefficient $c_{L}^{(3) q} \xi$ can be constrained at this level. ${ }^{10}$ For $c_{L}^{q}, c_{R}^{u}$ and $c_{R}^{d}$ the main constraints come from LEP1 measurements at the $Z$-pole. These can put bounds on deviations of the $Z$ couplings to quarks, $\delta g_{Z}^{q_{L, R}}$, and on $c_{L}^{q}$ and $c_{R}^{u, d}$.

As we saw, operators made of top quarks can depart from the MFV assumption due to the large top Yukawa coupling. If this is the case, we can still bound $\left(c_{L}^{q_{3}}+c_{L}^{(3) q_{3}}\right) \xi$ from the measurement of the $Z b_{L} \bar{b}_{L}$ coupling at LEP1 which also gives a per-mille bound. Interestingly, a $P_{L R}$ symmetry can be imposed in the BSM sector such that $c_{L}^{q_{3}}=-c_{L}^{(3) q_{3}}$ [see Eq. (C.10)], allowing for large deviations on $c_{L}^{q_{3}}-c_{L}^{(3) q_{3}}$. Recent LHC measurements of the $W t b$ coupling [24] put some bounds on $c_{L}^{(3) q_{3}}$ but they are not very strong. Also $c_{R}^{t}$ has practically no bound due to the large uncertainty in the determination of the $Z t_{R} \bar{t}_{R}$ coupling [25]. Bounds on the Wilson coefficient $c_{R}^{t b}$, see Eq. (17), arise from $b \rightarrow s \gamma$ and read $-0.001 \lesssim c_{R}^{t b} M_{W}^{2} / \Lambda^{2} \lesssim 0.006$ [26]. These bounds will be improved in the future by the LHC.

Four-fermion operators involving quarks, as those in the first box of Table 2, can also be constrained by recent LHC data [13], while the coefficients of the operators of the second box of Table 2 have no severe experimental constraints due to their Yukawa suppression. However, they can affect Higgs physics through operator mixing, as we will see in the next Section. Finally, bounds on dipole operators can be found, for example, in Ref. [5].

We conclude that, concerning the strength of experimental constraints, we can distinguish the following sets of $d=6$ operators:

(i) First, we have those which can only affect Higgs physics. We have $8+3$ operators of this

${ }^{10}$ The operator $\mathcal{O}_{L L}^{(3) q l}=\left(\bar{Q}_{L} \gamma_{\mu} \sigma^{a} Q_{L}\right)\left(\bar{L}_{L} \gamma^{\mu} \sigma^{a} L_{L}\right)$ also gives contributions to $\beta$-decays and $K$-decays, but this can be independently constrained by recent LHC data [23]. 
type (CP-even plus CP-odd respectively) for one family, with real coefficients given in Eq. (37) ${ }^{11}$. As shown in Section 3, they can independently modify the Higgs decaywidth to fermions, photons, gluons and $Z \gamma$, apart from a global rescaling of all Higgs amplitudes due to $c_{H}$.

(ii) A second set of operators are those whose coefficients are severely restricted by electroweak precision data, as explained above. Eliminating, by the EoM of Eq. (21), $\mathcal{O}_{2 B}, \mathcal{O}_{2 W}, \mathcal{O}_{2 G}$ and $\mathcal{O}_{L}^{l}, \mathcal{O}_{L}^{(3) l}$, these are $c_{W}+c_{B}$ and $c_{T}$ that affect the $W / Z$ propagator, and $c_{R}^{e}, c_{L}^{q}, c_{R}^{u, d}, c_{L}^{(3) q}$ that affect $V f \bar{f}$ vertices.

(iii) In a third set, we have the operator coefficients that can affect the $Z W W / \gamma W W$ vertices and are, at present, constrained at the few per-cent level. These are the combinations $\kappa_{H B}+\kappa_{H W}$ and $c_{W}+\kappa_{H W}$ (and also $c_{3 W}$ if we include $\lambda_{Z}$ in the analysis).

We finally would like to mention that our result is in contradiction with Ref. [27] that obtained a smaller number of parameters to characterize Higgs physics and triple gaugeboson vertices. The origin of this discrepancy is due to the following. In our basis it is clear that physics at LEP1 is not sensitive to the blind direction $c_{W}=-c_{B}$, since only the combination $c_{W}+c_{B}$ enters in the $\widehat{S}$ parameter. This blind direction, however, becomes more complicated when one goes to other bases, such as that of Ref. [9], in which $\mathcal{O}_{W}$ and $\mathcal{O}_{B}$ are eliminated [by using Eq. (21)] in favor of operators made of SM fermions. In such bases there is the risk of overestimating the number of independent experimental constraints on the Wilson coefficients.

\section{$5 \quad$ Running effects from $\Lambda$ to $M_{W}$}

So far, we have implicitly assumed that the Wilson coefficients were evaluated at the electroweak scale, at which their effects can be eventually measured. However, particular UV completions predict the values of those coefficients at the scale $\Lambda$ where the heavy BSM is integrated out. The RG evolution from $\Lambda$ down to the electroweak scale, described by the corresponding anomalous dimensions, can be important in many cases.

Our main interest is to calculate the anomalous dimensions of the Wilson coefficients that can have the largest impact on Higgs physics. As we explained in the previous section, these are the coefficients listed in Eq. (37). In Ref. [7] we already calculated the most relevant anomalous dimensions of the $\kappa_{i}$ in Eq. (37). We showed that tree-level Wilson coefficients do not enter, at the one-loop level, in the RGEs of the $\kappa_{i}$, a property that allowed us to complete the calculation of [6] for the anomalous dimensions relevant for $h \rightarrow \gamma \gamma, Z \gamma$. In this section we extend the analysis by calculating the anomalous dimensions for the 5 tree-level Wilson coefficients:

$$
\left\{c_{H}, c_{6}, c_{y_{t}}, c_{y_{b}}, c_{y_{\tau}}\right\}
$$

\footnotetext{
${ }^{11}$ If we relax the MFV assumption that the $c_{y_{f}}$ are real, in addition to the 3 operators $\operatorname{Re}\left(c_{y_{f}}\right)\left(\mathcal{O}_{y_{f}}+\mathcal{O}_{y_{f}}^{\dagger}\right)$ we should also consider the 3 CP-odd operators $\operatorname{Im}\left(c_{y_{f}}\right)\left(\mathcal{O}_{y_{f}}-\mathcal{O}_{y_{f}}^{\dagger}\right)$.
} 
We notice that even in the future, with better measurements of the Higgs couplings, and then better bounds on Eq. (51), we still expect Eq. (51) to give the main BSM contributions to Higgs physics, since other Wilson coefficients, such as $c_{W}$, are expected to receive even stronger constraints from LHC (for a given $\Lambda$ ).

Generically, the anomalous dimensions are functions of other Wilson coefficients:

$$
\gamma_{c_{i}}=\frac{d c_{i}}{d \log \mu}=\gamma_{c_{i}}\left(c_{j}\right)
$$

where $\mu$ is the renormalization scale. In the RHS of Eq. (52) we keep the $c_{j}$ coefficients that can potentially give the most significant contributions to the RG running. We keep the following $c_{j}$. First, those of Eq. (51) as they have no important experimental constraints and also are the most relevant in BSM scenarios with $g_{*}$ large. We also keep the Wilson coefficients of operators involving the top quark, departing from the MFV assumption. These are $\mathcal{O}_{L}^{q_{3}}, \mathcal{O}_{R}^{t}, \mathcal{O}_{L}^{(3) q_{3}}$ and $\mathcal{O}_{R}^{t b}$, in addition to the 4-fermion operators, $\mathcal{O}_{L L}^{q_{3}}, \mathcal{O}_{L L}^{(8) q_{3}}, \mathcal{O}_{L R}^{t}$, $\mathcal{O}_{L R}^{(8) t}, \mathcal{O}_{y_{t} y_{b}}, \mathcal{O}_{y_{t} y_{b}}^{(8)}, \mathcal{O}_{y_{t} y_{\tau}}$ and $\mathcal{O}_{y_{t} y_{\tau}}^{\prime}$. We have several motivations to keep them. First, they have no large constraints from experiments. Second, they can induce large effects on the anomalous dimensions of Eq. (51), since they are proportional to the top Yukawa coupling. Also their Wilson coefficients can be sizable in many BSM models, such as composite Higgs or supersymmetric theories, as we will discuss. To summarize, we consider in the RHS of Eq. (52) the following Wilson coefficients:

$$
\left\{c_{j}\right\}=\left\{c_{H}, c_{6}, c_{y_{t}}, c_{y_{b}}, c_{y_{\tau}}, c_{L}, c_{R}, c_{L}^{(3)}, c_{R}^{t b}, c_{L L}, c_{L L}^{(8)}, c_{L R}, c_{L R}^{(8)}, c_{y_{t} y_{b}}, c_{y_{t} y_{b}}^{(8)}, c_{y_{t} y_{\tau}}, c_{y_{t} y_{\tau}}^{\prime}\right\}
$$

where, from now on, we suppress the $q_{3}$ and $t$ superindices in the coefficients for simplicity.

We would like to mention that, even for those Wilson coefficients that receive experimental constraints, as those discussed in the previous section, the fact that the constraints apply to the ratios $c_{j} M_{W}^{2} / \Lambda^{2}$ means that bounds at the percent-level can allow for $c_{j} \sim O(1)$ if $\Lambda \sim O(\mathrm{TeV})$. These coefficients could then also give potentially non-negligible effects in the $\gamma_{c_{i}}$. An example of this is $c_{W}$. Nevertheless, one can still expect that the dominant effects will be given by the coefficients in Eq. (53) since, for a given $\Lambda$, they can always be larger than $c_{W}$.

In addition, we will also extend our calculation of anomalous dimensions to other Wilson coefficients beyond Eq. (51). These correspond to operators constrained by the present experimental data, and then their anomalous dimensions can be also useful to derive indirect bounds on the coefficients of Eq. (53). ${ }^{12}$

The anomalous dimensions presented below correspond to the basis of Tables 1 and 2, after using the five redundancies to eliminate the operators $\left\{\mathcal{O}_{L}^{l}, \mathcal{O}_{L}^{(3) l}, \mathcal{O}_{R R}^{e}, \mathcal{O}_{L L}^{l}, \mathcal{O}_{R R}^{(8) d}\right\}$. Nevertheless, removing or not these five operators and keeping the redundancy would not change our results (see Appendix B for more details).

\footnotetext{
12 Other anomalous dimensions were calculated in $[8,28]$.
} 


\subsection{Anomalous dimensions of operators relevant for Higgs physics}

We present here the anomalous dimensions for the Wilson coefficients in Eq. (51), the ones expected to dominate deviations in Higgs physics, including the effects from the Wilson coefficients in Eq. (53). These are given by

$$
\begin{aligned}
16 \pi^{2} \gamma_{c_{H}}= & {\left[4 N_{c} y_{t}^{2}+24 \lambda-\frac{3}{2}\left(3 g^{2}+2 g^{\prime 2}\right)\right] c_{H}+12 N_{c} y_{t}^{2} c_{L}^{(3)} } \\
16 \pi^{2} \gamma_{\lambda c_{6}}= & 6\left[N_{c} y_{t}^{2}+18 \lambda-\frac{3}{4}\left(3 g^{2}+g^{\prime 2}\right)\right] \lambda c_{6}+2\left(40 \lambda-3 g^{2}\right) \lambda c_{H} \\
& -16 N_{c} \lambda y_{t}^{2} c_{L}^{(3)}+8 N_{c} y_{t}^{2}\left(\lambda-y_{t}^{2}\right) c_{y_{t}}, \\
16 \pi^{2} \gamma_{c_{y_{t}}}= & {\left[\left(4 N_{c}+9\right) y_{t}^{2}+24 \lambda-\frac{3}{2}\left(3 g^{2}+g^{\prime 2}\right)\right] c_{y_{t}}+\left(3 y_{t}^{2}+2 \lambda-\frac{3}{2} g^{2}\right) c_{H} } \\
& +\left(2 y_{t}^{2}+4 \lambda-3 g^{2}-g^{\prime 2}\right) c_{R}-2\left(y_{t}^{2}+2 \lambda+2 g^{\prime 2}\right) c_{L} \\
& +4\left(-N_{c} y_{t}^{2}+3 \lambda+g^{\prime 2}\right) c_{L}^{(3)}+8\left(y_{t}^{2}-\lambda\right)\left[c_{L R}+C_{F} c_{L R}^{(8)}\right] \\
16 \pi^{2} \gamma_{c_{y_{b}}}= & {\left[2\left(N_{c}+1\right) y_{t}^{2}+24 \lambda-\frac{3}{2}\left(3 g^{2}+g^{\prime 2}\right)\right] c_{y_{b}}+\left(2 \lambda-\frac{3}{2} g^{2}\right) c_{H}+\left(2 N_{c}-1\right) y_{t}^{2} c_{y_{t}} } \\
& +2\left(2 \lambda+g^{\prime 2}\right) c_{L}+2\left[\left(3-2 N_{c}\right) y_{t}^{2}+6 \lambda+g^{\prime 2}\right] c_{L}^{(3)}-4 \frac{y_{t}^{2}}{g_{*}^{2}}\left(y_{t}^{2}+2 \lambda-\frac{3}{2} g^{2}\right) c_{R}^{t b} \\
& +2 \frac{y_{t}^{2}}{g_{*}^{2}}\left(\lambda-y_{t}^{2}\right)\left[\left(2 N_{c}+1\right) c_{y_{t} y_{b}}+C_{F} c_{y_{t} y_{b}}^{(8)}\right], \\
& -2 \frac{y_{t}^{2}}{g_{*}^{2}} N_{c}\left(\lambda-y_{t}^{2}\right)\left(2 c_{y_{t} y_{\tau}}+c_{y_{t} y_{\tau}}^{\prime}\right) \\
16 \pi^{2} \gamma_{c_{y_{\tau}}}= & {\left[2 N_{c} y_{t}^{2}+24 \lambda-\frac{3}{2}\left(3 g^{2}+g^{\prime 2}\right)\right] c_{y_{\tau}}+\left(2 \lambda-\frac{3}{2} g^{2}\right) c_{H}+2 N_{c} y_{t}^{2}\left[c_{y_{t}}-2 c_{L}^{(3)}\right] } \\
& {[57) } \\
= & (58) \\
= &
\end{aligned}
$$

where $N_{c}=3$ is the number of colors and $C_{F}=\left(N_{c}^{2}-1\right) /\left(2 N_{c}\right)$. Parametrically one has $\gamma_{c_{i}} \sim g_{j}^{2} c_{j} / 16 \pi^{2}$ and we only keep $g_{j}^{2}=\left\{y_{t}^{2}, g_{s}^{2}, g^{2}, g^{\prime 2}, \lambda\right\}$, dropping $g_{j}^{2}=\left\{y_{b}^{2}, y_{\tau}^{2}, \ldots\right\}$. We remark that, to calculate these anomalous dimensions, one has to take into account that redundant operators removed from our operator basis are nevertheless generated through renormalization at the one-loop level. For details about how to deal with this effect, see Appendices A and B. The need to care about such effect also means that the RGEs depend on the choice of redundant operators (i.e. on the basis).

Let us make a quantitative analysis of the size of these radiative effects. Working at one-loop leading log order,

$$
c_{i}\left(M_{t}\right) \simeq c_{i}(\Lambda)-\gamma_{c_{i}} \log \frac{\Lambda}{M_{t}},
$$

which is enough if we take $\Lambda \sim 2 \mathrm{TeV}$ as UV scale and $M_{t}$ as electroweak scale, we obtain 
the following radiative modifications of the Wilson coefficients, $\Delta c_{i} \equiv c_{i}\left(M_{t}\right)-c_{i}(2 \mathrm{TeV})$ :

$$
\begin{aligned}
\Delta c_{H}= & -0.17 c_{H}-0.49 c_{L}^{(3)}, \\
\Delta \lambda c_{6}= & -0.36 \lambda c_{6}-0.015 c_{H}+0.082 c_{L}^{(3)}+0.244 c_{y_{t}}, \\
\Delta c_{y_{t}}= & -0.30 c_{y_{t}}-0.035 c_{H}-0.013 c_{R}+0.043 c_{L}+0.13 c_{L}^{(3)}-0.093 c_{L R}-0.12 c_{L R}^{(8)}, \\
\Delta c_{y_{b}}= & -0.12 c_{y_{b}}-0.068 c_{y_{t}}+0.0060 c_{H}-0.012 c_{L}+0.054 c_{L}^{(3)}+0.027 c_{R}^{t b} / g_{*}^{2} \\
& +\left(0.16 c_{y_{t} y_{b}}+0.027 c_{y_{t} y_{b}}^{(8)}\right) / g_{*}^{2}, \\
\Delta c_{y_{\tau}}= & -0.096 c_{y_{\tau}}-0.081 c_{y_{t}}+0.0060 c_{H}+0.16 c_{L}^{(3)}+\left(0.012 c_{y_{t} y_{\tau}}+0.061 c_{y_{t} y_{\tau}}^{\prime}\right) / g_{*}^{2} .(60)
\end{aligned}
$$

We see that in a few cases, the numerical impact of operator mixing can be significant, like the mixing of $c_{L}^{(3)}$ into $c_{H} ; \lambda c_{6}$ and $c_{y_{t}}$ into $\lambda c_{6}$; and $c_{y_{t}}$ into itself.

\subsection{Anomalous dimensions of constrained operators}

Other interesting anomalous dimensions to calculate correspond to operators that are at present constrained by experiments. Here we present those of $c_{T}, c_{B}, c_{W}$, and for the top quark, $c_{R}, c_{L}$, and $c_{L}^{(3)}$ :

$$
\begin{aligned}
16 \pi^{2} \gamma_{c_{T}}= & \frac{3}{2} g^{\prime 2} c_{H}+4 N_{c} y_{t}^{2}\left(c_{R}-c_{L}\right) \\
16 \pi^{2} \gamma_{c_{R}}= & {\left[2\left(4+N_{c}\right) y_{t}^{2}-9 g^{2}-\frac{7}{3} g^{\prime 2}\right] c_{R}-4\left(N_{c}+1\right)\left(y_{t}^{2}-\frac{2}{9} g^{\prime 2}\right) c_{R R} } \\
& +2 N_{c}\left(y_{t}^{2}+\frac{1}{9} g^{\prime 2}\right) c_{L R}+2 y_{t}^{2}\left(\frac{1}{4} c_{H}-c_{L}\right) \\
16 \pi^{2} \gamma_{c_{L}}= & {\left[2\left(2+N_{c}\right) y_{t}^{2}-9 g^{2}-\frac{7}{3} g^{\prime 2}\right] c_{L}+2\left(y_{t}^{2}+\frac{1}{9} g^{\prime 2}\right)\left[\left(2 N_{c}+1\right) c_{L L}+C_{F} c_{L L}^{(8)}\right] } \\
& -2 N_{c}\left(y_{t}^{2}-\frac{2}{9} g^{\prime 2}\right) c_{L R}-y_{t}^{2}\left(\frac{1}{4} c_{H}+c_{R}+9 c_{L}^{(3)}\right) \\
16 \pi^{2} \gamma_{c_{L}^{(3)}}= & {\left[2\left(1+N_{c}\right) y_{t}^{2}-\frac{16}{3} g^{2}-3 g^{\prime 2}\right] c_{L}^{(3)}-2\left(y_{t}^{2}-\frac{1}{3} g^{2}\right)\left[c_{L L}+C_{F} c_{L L}^{(8)}\right] } \\
& +y_{t}^{2}\left(\frac{1}{4} c_{H}-3 c_{L}\right) \\
16 \pi^{2} \gamma_{c_{W}}= & \frac{1}{3} g_{*}^{2}\left[16 N_{c} c_{L}^{(3)}-c_{H}\right] \\
16 \pi^{2} \gamma_{c_{B}}= & \frac{1}{3} g_{*}^{2}\left[\frac{8}{3} N_{c}\left(2 c_{R}+c_{L}\right)-c_{H}\right] .
\end{aligned}
$$

From them we can calculate the leading-log corrections to $c_{B}+c_{W}, c_{T}$ and $c_{L}+c_{L}^{(3)}$ that are highly constrained by $\widehat{S}, \widehat{T}$ and the $Z b b$-coupling, as has been discussed in Section 4 . In this 
way, coefficients that are more loosely constrained by direct processes, such as $c_{H}, c_{L}$ or $c_{R}$, can get indirect bounds from LEP1 and Tevatron measurements.

Integrating the RGEs of Eq. (66), at the one-loop leading-log order, between the cutoff scale $\Lambda=2 \mathrm{TeV}$ and the electroweak scale, that we take here $M_{t}$, one gets ${ }^{13}$

$$
\begin{aligned}
\Delta \widehat{T} & =\Delta c_{T} \xi=\left[-0.003 c_{H}+0.16\left(c_{L}-c_{R}\right)\right] \xi \\
\Delta \widehat{S} & =\Delta\left(c_{B}+c_{W}\right) \frac{M_{W}^{2}}{\Lambda^{2}}=\left[0.001 c_{H}-0.01 c_{R}-0.004 c_{L}-0.03 c_{L}^{(3)}\right] \xi, \\
\Delta \frac{\delta g_{Z}^{b_{L}}}{g_{Z}^{b_{L}}} & =\frac{\Delta\left[c_{L}+c_{L}^{(3)}\right]}{1-(2 / 3) \sin ^{2} \theta_{W}} \xi \simeq \Delta\left[c_{L}+c_{L}^{(3)}\right] \xi \\
& =\left[0.01 c_{R}-0.03 c_{L}+0.06 c_{L}^{(3)}-0.17 c_{L L}-0.0064 c_{L L}^{(8)}+0.08 c_{L R}\right] \xi,
\end{aligned}
$$

where $\Delta c_{i} \equiv c_{i}\left(M_{t}\right)-c_{i}(2 \mathrm{TeV})$. Notice that even if a $P_{L R}$ symmetry of the BSM sector enforces $c_{L}+c_{L}^{(3)}=0$, we can have a nonzero $c_{L}+c_{L}^{(3)}$ from the RG running, since the SM does not respect this parity. The fact that the three quantities above are constrained at the per-mille level implies that the top coefficients, $\left\{c_{L}, c_{R}, \ldots\right\} \times \xi$ cannot be of order one. Obviously, we are barring the possibility of cancellations between the initial value of the Wilson coefficients at the scale $\Lambda$ and the radiative effects $\sim \gamma_{c_{i}} \log \left(\Lambda / M_{t}\right)$, that could only be possible by accident.

\section{$6 \quad$ RGE impact on the predictions of Wilson coefficients}

Here we want to study the impact of the evolution of the Wilson coefficients from the UV scale $\Lambda$ down to the electroweak scale at which they affect Higgs physics. This running can modify the predictions arising from BSM models. We present three examples: two-Higgs doublet models $(2 \mathrm{HDM})$, universal theories, and scenarios with sizeable $c_{L, R}$, such as composite-top models.

2HDM and Supersymmetric theories: At tree-level, assuming ordinary $R$-parity, the only $d=6$ operators that can be induced in supersymmetric models arise from the exchange of the extra Higgses since these are the only $R$-even heavy fields. In particular, the MSSM contains an extra heavy Higgs doublet. It is therefore well motivated to look for the impact of an extra heavy Higgs doublet in SM Higgs physics.

Denoting the heavy Higgs by $H^{\prime}$, defined to have $Y_{H^{\prime}}=1 / 2$, its relevant couplings to the SM fermions and Higgs are given by

$$
\mathcal{L}^{\prime}=-\alpha_{u} y_{u} \bar{Q}_{L} \widetilde{H}^{\prime} u_{R}-\alpha_{d} y_{b} \bar{Q}_{L} H^{\prime} d_{R}-\alpha_{e} y_{e} \bar{l}_{L} H^{\prime} e_{R}-\lambda^{\prime} H^{\prime \dagger} H|H|^{2}+\text { h.c. }+\cdots
$$

where $\alpha_{u, d, e}$ are constants and we assume that $\lambda^{\prime}$ is a real number. In particular 2HDMs,

\footnotetext{
13 The effects of $c_{H}$ and those of $c_{L, R}$ on $\widehat{T}$ were already calculated in [29] and [30] respectively.
} 
these constants are

$$
\begin{array}{ll}
\alpha_{u}=\alpha_{d}=\alpha_{e}=\tan \beta, & \text { for type-I 2HDM } \\
\alpha_{u}=-\cot \beta, \quad \alpha_{d}=\alpha_{e}=\tan \beta, & \text { for type-II 2HDM (MSSM) }
\end{array}
$$

where $\tan \beta$ defines the rotation from the original basis, in which only one Higgs couples to a given type of fermion, to the mass-eigenstate basis before EWSB. At the order we work $\left(\sim v^{2} / \Lambda^{2}\right), \tan \beta$ coincides with that defined in the MSSM. Integrating out this doublet at tree-level, we obtain the following nonzero coefficients for the third-family $d=6$ operators:

$$
\begin{aligned}
& g_{*}^{2} c_{y_{t}}=\alpha_{t} \lambda^{\prime}, \quad g_{*}^{2} c_{y_{b}}=\alpha_{b} \lambda^{\prime}, \quad g_{*}^{2} c_{y_{\tau}}=\alpha_{\tau} \lambda^{\prime}, \quad g_{*}^{2} \lambda c_{6}=\lambda^{\prime 2} \\
& g_{*}^{2} c_{L R}^{(8)}=2 N_{c} g_{*}^{2} c_{L R}=-\alpha_{t}^{2} y_{t}^{2}, \quad c_{y_{t} y_{b}}=\alpha_{t} \alpha_{b}, \quad c_{y_{t} y_{\tau}}=\alpha_{t} \alpha_{\tau} .
\end{aligned}
$$

We have used $\left(\bar{Q}_{L} t_{R}\right)\left(\bar{t}_{R} Q_{L}\right)=-\left(\bar{Q}_{L} T^{A} \gamma^{\mu} Q_{L}\right)\left(\bar{t}_{R} T^{A} \gamma_{\mu} t_{R}\right)-\left(\bar{Q}_{L} \gamma^{\mu} Q_{L}\right)\left(\bar{t}_{R} \gamma_{\mu} t_{R}\right) /\left(2 N_{c}\right)$ and now $\Lambda=M_{H^{\prime}}$. Under the RGE flow of Eqs. (56)-(58) the operators $\mathcal{O}_{y_{f}}$ mix with $\mathcal{O}_{L R}, \mathcal{O}_{L R}^{(8)}$, $\mathcal{O}_{y_{u} y_{d}}$ and $\mathcal{O}_{y_{u} y_{\tau}}$. In the type-II $2 \mathrm{HDM}$, we obtain in the one-loop leading-log approximation and neglecting $O\left(\lambda, g^{2}, g^{2}\right)$ corrections:

$$
\begin{aligned}
& g_{*}^{2} c_{y_{t}}\left(m_{h}\right)=-\frac{\lambda^{\prime}}{t_{\beta}}\left[1-\frac{21 y_{t}^{2}}{16 \pi^{2}} \log \frac{M_{H^{\prime}}}{m_{h}}\right]+\frac{3 y_{t}^{4}}{4 \pi^{2} t_{\beta}^{2}} \log \frac{M_{H^{\prime}}}{m_{h}}, \\
& g_{*}^{2} c_{y_{b}}\left(m_{h}\right)=\lambda^{\prime} t_{\beta}\left[1-\frac{y_{t}^{2}}{2 \pi^{2}} \log \frac{M_{H^{\prime}}}{m_{h}}\right]+\frac{y_{t}^{2}}{16 \pi^{2}}\left[5 \frac{\lambda^{\prime}}{t_{\beta}}-14 y_{t}^{2}\right] \log \frac{M_{H^{\prime}}}{m_{h}}, \\
& g_{*}^{2} c_{y_{\tau}}\left(m_{h}\right)=\lambda^{\prime} t_{\beta}\left[1-\frac{3 y_{t}^{2}}{8 \pi^{2}} \log \frac{M_{H^{\prime}}}{m_{h}}\right]+\frac{3 y_{t}^{2}}{8 \pi^{2}}\left[\frac{\lambda^{\prime}}{t_{\beta}}-2 y_{t}^{2}\right] \log \frac{M_{H^{\prime}}}{m_{h}}
\end{aligned}
$$

with $t_{\beta} \equiv \tan \beta$.

To illustrate the impact of these radiative effects, let us consider the MSSM, a model which predicts $\lambda^{\prime}=(1 / 8)\left(g^{2}+g^{\prime 2}\right) \sin 4 \beta$ at tree-level [31]. We take the stop mass scale $M_{\tilde{t}}$ large enough to get $m_{h} \simeq 125 \mathrm{GeV}$ through the well-known loop corrections to the Higgs quartic coupling, which at one-loop and zero stop mixing read:

$$
\lambda\left(m_{h}\right)=\frac{1}{8}\left(g^{2}+g^{\prime 2}\right) \cos ^{2} 2 \beta+\frac{3 y_{t}^{4}}{16 \pi^{2}} \log \frac{M_{\tilde{t}}^{2}}{M_{t}^{2}},
$$

which is precise enough for our illustrative purposes. For consistency we must also include similar radiative corrections to $\lambda^{\prime}$, which read at one-loop:

$$
\lambda^{\prime}\left(M_{H^{\prime}}\right)=\frac{1}{8}\left(g^{2}+g^{\prime 2}\right) \sin 4 \beta-\frac{3 y_{t}^{4}}{8 \pi^{2} t_{\beta}} \log \frac{M_{\tilde{t}}^{2}}{M_{H^{\prime}}^{2}} .
$$

This gives the value of $\lambda^{\prime}$ that we can then plug in Eq. (74) to obtain the RG-improved corrections for $g_{h f f}$ induced by integrating out the heavy Higgses. The result is shown as a function of $t_{\beta}$ in Fig. 1, which compares the tree-level result (dashed lines) and the oneloop result (solid lines) which takes into account the running from $\Lambda=M_{H^{\prime}}$ down to the 

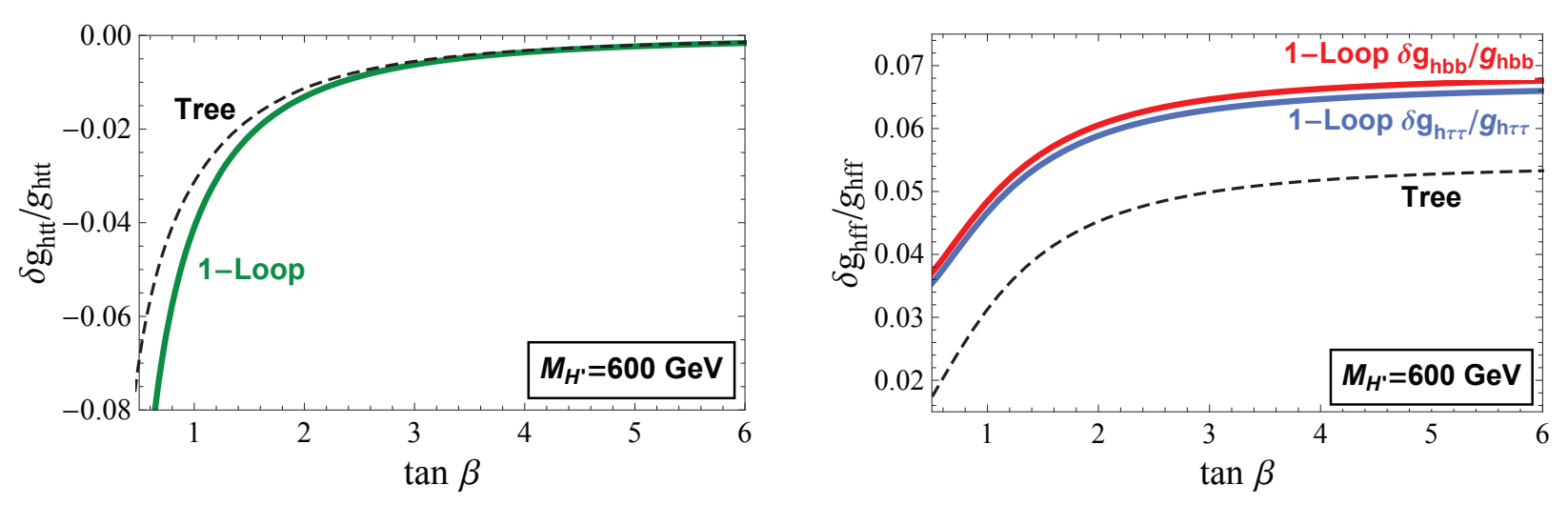

Figure 1: Relative modification of the Higgs coupling to fermions, $\delta g_{h f f} / g_{h f f}=-c_{y_{f}} \xi$, Eq. (26), at tree-level (dashed line) and after including RGE effects from $\Lambda$ to the electroweak scale (solid lines) as a function of $\tan \beta$ in an $M S S M$ scenario with $\Lambda=M_{H^{\prime}}=600$ GeV and unmixed stops heavy enough to reproduce $m_{h}=125 \mathrm{GeV}$. Left plot: top coupling. Right plot: bottom (lower solid line) and tau (upper solid line) couplings.

electroweak scale $m_{h}$. One sees that the effect of the running can be quite significant, easily $\sim 50 \%$ or more. The importance of this effect can be further appreciated in Fig. 2, which shows the lower bound one could set on $M_{H^{\prime}}$ from an upper bound on $\delta g_{h b b} / g_{h b b}$, the deviation of $g_{h b b}$ from its SM value. By comparing the tree-level bound (dashed line) and the one-loop bound (solid line) one sees that the bound is shifted significantly by the inclusion of the RG corrections from $M_{H^{\prime}}$ to $m_{h}$.

Finally, notice that $c_{H}$, which is not generated in the MSSM at tree-level since there are no heavy $R$-even singlet states, is not generated by the RGE evolution and therefore is also zero in the leading-log approximation.

Universal theories and composite Higgs models: Universal theories predict $c_{y_{u}}=c_{y_{d}}=$ $c_{y_{e}}$. This prediction is modified by the evolution of these coefficients from the scale $\Lambda$, where they are generated, down to the electroweak scale. In particular, for $\Lambda=2 \mathrm{TeV}$, we find that the breaking of universality due to the top Yukawa coupling gives

$$
\begin{aligned}
& c_{y_{t}}\left(m_{h}\right)=c_{y_{b}}\left(m_{h}\right)\left(1-\frac{8 y_{t}^{2}}{16 \pi^{2}} \log \frac{\Lambda}{m_{h}}\right)-\frac{3 y_{t}^{2} c_{H}}{16 \pi^{2}} \log \frac{\Lambda}{m_{h}} \simeq 0.88 c_{y_{b}}\left(m_{h}\right)-0.05 c_{H}, \\
& c_{y_{b}}\left(m_{h}\right)=c_{y_{\tau}}\left(m_{h}\right)\left(1-\frac{y_{t}^{2}}{16 \pi^{2}} \log \frac{\Lambda}{m_{h}}\right) \simeq 0.98 c_{y_{\tau}}\left(m_{h}\right) .
\end{aligned}
$$

This is a sizeable departure from universality for $c_{y_{t}}$ that will have to be taken into account when fitting these models to data. Also it is worth noticing that in models in which only $c_{H}$ is generated (models with only heavy singlets) and $c_{y_{f}}(\Lambda)=0$, the value of $c_{y_{f}}$ is also very 


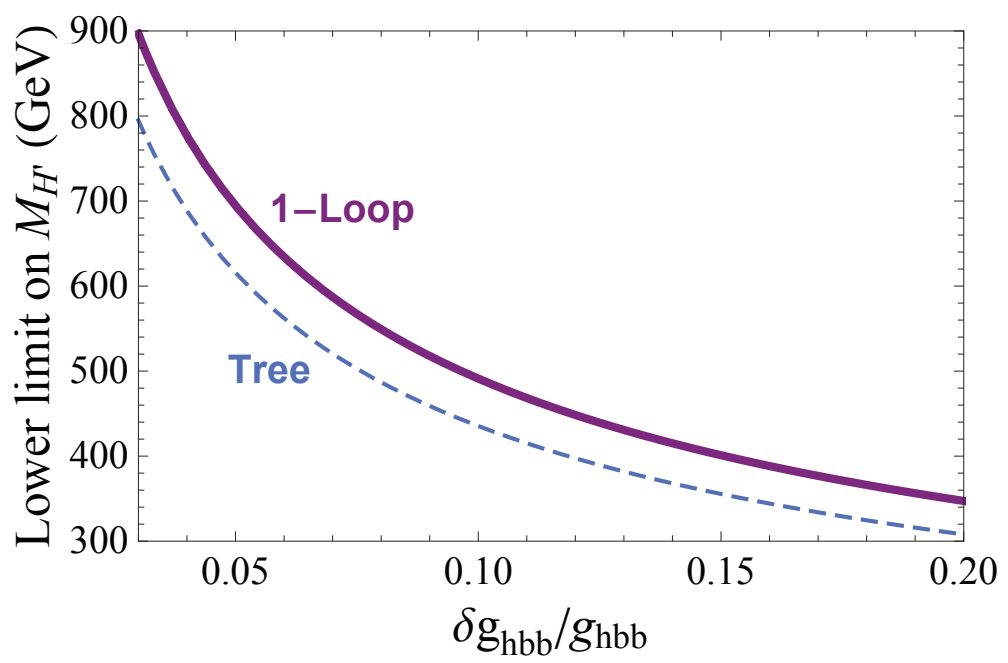

Figure 2: Lower bound on $M_{H^{\prime}}$ as a function of the upper bound on the relative deviation $\delta g_{h b b} / g_{h b b}$, in an MSSM scenario with $\tan \beta=5$ and unmixed stops heavy enough to reproduce $m_{h}=125 \mathrm{GeV}$. The dashed line corresponds to a tree-level analysis (parameters calculated at the scale $M_{H^{\prime}}$ ), while the solid line includes the $R G$ running from $M_{H^{\prime}}$ down to $m_{h}$.

small at low-energies, $c_{y_{f}}\left(m_{h}\right) \simeq 0$. In the minimal composite Higgs model, we also have the prediction $c_{H}=1$ at $\Lambda \sim 2 \mathrm{TeV}$ [4]. We find that the RG effects give a $\sim 20 \%$ reduction of this prediction.

Models with a non-SM top: The top is the only quark whose properties are not yet measured at high precision, allowing then sizeable deviations from their SM predictions. There are also theoretical motivations to expect the top to be the quark with the largest deviations from the SM predictions, as it is the quark with the largest coupling to the Higgs. This is specially true in composite Higgs models where one expects the top to show also certain degree of compositeness. In these examples we can expect sizable values for $c_{R}, c_{L}^{(3)}, c_{L}$ and $c_{L R}$ that can affect, at the one loop-level, the Higgs coefficients $c_{H}$ and $c_{y_{f}}$. As it is clear from Eq. (60) the effects of $c_{R}$ on the RGE evolution of $c_{H}$ and $c_{y_{f}}$ are very small. Nevertheless, those from $c_{L}^{(3)}$ and $c_{L}$ are quite sizeable, even in the limit $c_{L} \simeq-c_{L}^{(3)}$ as required in order to avoid large tree-level contributions to $Z b \bar{b}$. Unfortunately these coefficients also give large one-loop effects to the $\widehat{T}$ and $\widehat{S}$ parameters and $Z b b$, as Eqs. (67)-(69) show, that bounds them to be small (unless $\xi$ is small). Interestingly, the coefficient $c_{L R}^{(8)}$ is not constrained by Eqs. (67)-(69). Therefore it can give sizeable contributions to the RGE evolution of $c_{y_{t}}$ :

$$
c_{y_{t}}\left(m_{h}\right)=c_{y_{t}}(\Lambda)-\frac{2 y_{t}^{2}}{3 \pi^{2}} c_{L R}^{(8)} \log \frac{\Lambda}{m_{h}},
$$

that is of order $\sim 15 \%$. A nonzero $c_{L R}^{(8)}$ could arise from integrating out a massive gluon 
coupled to the top.

\section{Conclusions}

As the measurements of the Higgs properties improve, it will be important to understand their implications for BSM models. In this article we have adopted the framework of effective Lagrangians as a tool to study the effects of $d=6$ operators in Higgs physics. As a first step, we have discussed the choice of basis of operators. Our basis has been defined following $[4,7]$ that distinguished two classes of operators: tree-level (or current-current) operators, and one-loop operators. This choice can be important when calculating one-loop operator mixing, since most of the tree-level operators do not mix with one-loop operators under RG evolution [7]. Another important property of our basis is that it contains a subset of $5 \mathrm{CP}$ even operators made of Higgs and gauge field-strengths, that in our case are $\mathcal{O}_{W, B} \mathcal{O}_{H W, H B}$ and $\mathcal{O}_{B B}$, (leaving aside $\mathcal{O}_{G G}$ ). We have found that it is important to keep these 5 operators to make the connection with experiments more transparent [these subset could also be written with $\mathcal{O}_{W B, W W}$ by using the identities (12) and (13)]. Bases, such as [9] and [27], that eliminate two of these operators in favor of operators made of SM fermions, as it can be done by using the EoM, have dangerous blind directions for LEP1 experiments, which make the contact with experiments more difficult.

We have calculated the modifications that the operators of the effective Lagrangian induce in the Higgs couplings relevant for the main decays and production mechanisms. It has been shown that these operators can be divided in two subsets. There are 11 operators (for one family) with coefficients given in Eq. (37), that can only affect Higgs physics and no other SM processes at tree-level. The number 11 can be deduced from counting the number of independent operators one can write as $|H|^{2} \mathcal{O}_{4}$ with $\mathcal{O}_{4}$ a $d=4$ operator formed with SM fields. The second subset, formed by the rest of operators, enter in other SM processes and therefore can be constrained by non-Higgs experiments. Among the latter, considering only the CP-even ones, we have found that the least constrained correspond to the two combinations of Wilson coefficients appearing in the measurements of the $Z W W / \gamma W W$ coupling, Eq. (48), that LEP2 has only constrained at the few per-cent level. LHC will probe these vertices with better accuracy, so that it will be able to improve these constraints.

We have calculated the anomalous dimensions of the 5 tree-level operators of the list Eq. (37), which allows us to calculate the running of the coefficients from the high-energy scale $\Lambda$ where they are generated down to the electroweak scale. All technical details of these calculations have been discussed in Appendix B. Since $\widehat{S}$ and $\widehat{T}$ parameters, and the $Z b \bar{b}$ coupling are very well constrained, we have also calculated the anomalous dimension of the operators contributing to these quantities. In this way, we can put indirect bounds on Higgs operators.

We have applied our results to BSM models as MSSM, universal theories (as composite Higgs models) and models with non-standard top couplings. In such models we have evaluated 
the leading-log corrections to the predictions for the Higgs couplings. The corrections from the running can be quite large for $\Lambda \sim$ few TeV, as Fig. 2 shows. Our calculation of the anomalous dimensions is an aspect of the physics of the $d=6$ operators which will become more relevant as soon as we have better measurements of the Higgs couplings.

\section{Acknowledgements}

We thank M. Carrel, C. Grojean, M. Montull, F. Riva and A. Wulzer for useful discussions. J.R.E. thanks CERN for hospitality and partial financial support during some stages of this work. This work has been partly supported by Spanish Consolider Ingenio 2010 Programme CPAN (CSD2007-00042) and the Spanish Ministry MICNN under grants FPA2010-17747 and FPA2011-25948; and the Generalitat de Catalunya grant 2009SGR894. The work of A.P. has also been supported by the ICREA Academia Program. The work of J.E.M. has been supported by the Spanish Ministry MECD through the FPU grant AP2010-3193. 


\section{A Currents, redundant operators and field shifts}

In this Appendix we first list, in Subsection A.1, the different currents (of dimension $\leq 3$ ) built from SM fields that enter into the $d=6$ current-current operators. We examine in Subsection A.2 how these operators can be generated from integrating out heavy particles discussing what type of operators appear depending on the quantum numbers of the heavy fields. Some of these operators are redundant and can be eliminated from the Lagrangian by using the field equations of motion or, equivalently, by field redefinitions. We discuss this point in Subsection A.3, where we give a possible set of field redefinitions that can be used to get rid of the redundant operators.

\section{A.1 Currents of SM fields}

For simplicity we limit our examples of currents to the SM with a single family of fermions, the generalization to 3 families being straightforward. The scalar currents are:

$$
\begin{array}{lll}
J_{H}=|H|^{2}, & J_{H}^{(2)}=H|H|^{2}, & J_{\square H}=D_{\mu}^{2} H, \\
J_{H}^{a}=H^{\dagger} \sigma^{a} H, & J_{y_{f} H}=y_{f} \bar{F}_{L} f_{R}, & J_{y_{f} H}^{A}=y_{f} \bar{Q}_{L} T^{A} f_{R},
\end{array}
$$

where $T^{A}$ are the $S U(3)_{c}$ generators and from now on we use the notation $F_{L}=\left\{Q_{L}, L_{L}\right\}$ and $f_{R}=\left\{u_{R}, d_{R}, e_{R}\right\}$ for fields, while $F=\{q, l\}$ and $f=\{u, d, e\}$ are used for the corresponding operator indices. Obviously, one can also have the conjugate currents: $\tilde{J}_{H}^{(2)}=\tilde{H}|H|^{2}, \tilde{J}_{\square H}=$ $D_{\mu}^{2} \tilde{H}$, etc.

There are also vector currents made of SM bosons, like:

$$
\begin{array}{lll}
J_{H}^{\mu}=i H^{\dagger} \stackrel{\leftrightarrow}{D^{\mu}} H, & J_{W_{R}}^{\mu}=i \widetilde{H}^{\dagger} \stackrel{\leftrightarrow}{D^{\mu}} H, & J_{H}^{a \mu}=i H^{\dagger} \sigma^{a} \stackrel{\leftrightarrow}{D^{\mu}} H \\
J_{B}^{\mu}=\partial_{\nu} B^{\mu \nu}, & J_{W}^{a \mu}=D_{\nu} W^{a \mu \nu}, & J_{G}^{A \mu}=D_{\nu} G^{A \mu \nu}
\end{array}
$$

and made of SM fermions, like:

$$
\begin{array}{ll}
J_{f f^{\prime}}^{\mu}=\bar{f}_{R} \gamma^{\mu} f_{R}^{\prime}, & J_{F}^{\mu}=\bar{F}_{L} \gamma^{\mu} F_{L}, \quad J_{F}^{a \mu}=\bar{F}_{L} \sigma^{a} \gamma^{\mu} F_{L}, \\
J_{f}^{A \mu}=\bar{f}_{R} T^{A} \gamma^{\mu} f_{R}, & J_{Q}^{A \mu}=\bar{Q}_{L} T^{A} \gamma^{\mu} Q_{L},
\end{array}
$$

as well as the lepto-quark currents:

$$
J_{Q e}^{\alpha}=y_{e} \bar{Q}_{L}^{\alpha} e_{R}, \quad J_{L u}^{\alpha}=y_{u} \bar{L}_{L} u_{R}^{\alpha}, \quad J_{L d}^{\alpha}=y_{d} \bar{L}_{L} d_{R}^{\alpha},
$$

where we write explicitly the color index $\alpha$. Finally, we list fermionic currents made of SM fields. They can be $S U(2)_{L}$ singlets:

$$
J_{D f}=i \not D f_{R}, \quad J_{y_{f} \bar{f}_{R}}=\left\{y_{u}^{\dagger} \widetilde{H}^{\dagger} Q_{L}, y_{d}^{\dagger} H^{\dagger} Q_{L}, y_{e}^{\dagger} H^{\dagger} L_{L}\right\}
$$

doublets:

$$
J_{D F}=i \not D F_{L}, \quad J_{y_{f} \bar{F}_{L}}=\left\{y_{u} \widetilde{H} u_{R}, y_{d} H d_{R}, y_{e} H e_{R}\right\}
$$


or triplets:

$$
J_{\widetilde{H} F}^{a}=\left\{\widetilde{H}^{\dagger} \sigma^{a} Q_{L}, \widetilde{H}^{\dagger} \sigma^{a} L_{L}\right\}, \quad J_{H F}^{a}=\left\{H^{\dagger} \sigma^{a} Q_{L}, H^{\dagger} \sigma^{a} L_{L}\right\} .
$$

The previous list of SM currents is not complete but contains all the currents necessary to build the current-current operators of our basis (defined in the main body of the paper), as well as many of the redundant operators.

\section{A.2 Current-current operators}

The $d=6$ current-current operators can in principle be generated from the tree-level exchange of heavy fields. We can then classify such operators by the quantum numbers of the exchanged heavy fields. We present such classification below (giving explicit expressions for those redundant operators that appear here for the first time.). Finding possible deformations in SM couplings that can be assigned to particular current-current operators can offer crucial information in identifying the heavy physics responsible for such effects.

○ Scalar $\times$ scalar

The exchange of a heavy scalar singlet can lead (after integration by parts) to:

$$
-J_{H} \square J_{H}=2 \mathcal{O}_{H} \text {. }
$$

From a heavy scalar $S U(2)_{L}$-doublet we get:

$$
\begin{array}{ll}
\lambda J_{H}^{(2) \dagger} J_{H}^{(2)}=\mathcal{O}_{6}, & J_{H}^{(2) \dagger} J_{\square H}+\text { h.c. }=-2\left(\mathcal{O}_{H}+\mathcal{O}_{r}\right), \\
J_{y_{u} H} \tilde{J}_{H}^{(2)}=\mathcal{O}_{y_{u}}, & J_{y_{d} H}^{\dagger} J_{y_{e} H}=\mathcal{O}_{y_{d} y_{e}}, \\
\left(J_{y_{u} H}\right)^{r} \epsilon_{r s}\left(J_{y_{e} H}\right)^{s}=\mathcal{O}_{y_{u} y_{e}}, & \left(J_{y_{u} H}\right)^{r} \epsilon_{r s}\left(J_{y_{d} H}\right)^{s}=\mathcal{O}_{y_{u} y_{d}},
\end{array}
$$

and also:

$$
\begin{aligned}
& J_{\square H}^{\dagger} J_{\square H}=\left|D_{\mu}^{2} H\right|^{2} \equiv \mathcal{O}_{K 4}, \\
& J_{y_{u} H} \tilde{J}_{\square H}=-y_{u} D_{\mu}\left(\bar{Q}_{L} u_{R}\right) D^{\mu} \widetilde{H} \equiv-\mathcal{O}_{y H}^{u} .
\end{aligned}
$$

If the heavy doublet is also charged under $S U(3)_{c}$ we can get:

$$
\left(J_{Q e}^{\alpha}\right)^{r} \epsilon_{r s}\left(J_{L u}^{\alpha}\right)^{s}=\mathcal{O}_{y_{u} y_{e}}^{\prime}, \quad\left(J_{y_{u} H}^{A}\right)^{r} \epsilon_{r s}\left(J_{y_{d} H}^{A}\right)^{s}=\mathcal{O}_{y_{u} y_{d}}^{(8)},
$$

while, from a heavy scalar $S U(2)_{L}$-triplet we would obtain:

$$
J_{H}^{a} D^{2} J_{H}^{a}=-2 \mathcal{O}_{T}-4 \mathcal{O}_{r} .
$$

\section{- Vector $\times$ vector}

From the exchange of a heavy singlet vector one can get:

$$
\begin{aligned}
& J_{H}^{\mu} J_{H \mu}=-2 \mathcal{O}_{T}, \quad g^{\prime} J_{B}^{\mu} J_{H \mu}=2 \mathcal{O}_{B}, \quad J_{u u}^{\mu} J_{H \mu}=\mathcal{O}_{R}^{u}, \\
& J_{H}^{\mu} J_{F \mu}=\mathcal{O}_{L}^{F}, \quad J_{B}^{\mu} J_{B \mu}=-2 \mathcal{O}_{2 B}, \quad J_{u u}^{\mu} J_{u u \mu}=\mathcal{O}_{R R}^{u}, \\
& J_{u u}^{\mu} J_{F \mu}=\mathcal{O}_{L R}^{u}, \quad J_{F}^{\mu} J_{F \mu}=\mathcal{O}_{L L}^{F}, \quad y_{u}^{\dagger} y_{d} J_{W_{R} \mu} J_{u d}^{\mu}=\mathcal{O}_{R}^{u d},
\end{aligned}
$$


as well as

$$
\begin{aligned}
g^{\prime} J_{B}^{\mu} J_{u u \mu} & =g^{\prime}\left(\bar{u}_{R} \gamma^{\mu} u_{R}\right)\left(\partial^{\nu} B_{\mu \nu}\right) \equiv \mathcal{O}_{B R}^{u}, \\
g^{\prime} J_{B}^{\mu} J_{F \mu} & =g^{\prime}\left(\bar{F}_{L} \gamma^{\mu} F_{L}\right)\left(\partial^{\nu} B_{\mu \nu}\right) \equiv \mathcal{O}_{B L}^{F} .
\end{aligned}
$$

The exchange of a heavy $S U(2)_{L}$-triplet vector can produce:

$$
\begin{aligned}
& J_{H}^{a \mu} J_{H \mu}^{a}=-2 \mathcal{O}_{H}+4 \mathcal{O}_{r}, \quad g J_{W \mu}^{a} J_{H}^{a \mu}=2 \mathcal{O}_{W}, \quad J_{F}^{a \mu} J_{H \mu}^{a}=\mathcal{O}_{L}^{(3) F}, \\
& J_{W}^{a \mu} J_{W \mu}^{a}=-2 \mathcal{O}_{2 W}, \quad J_{L \mu}^{a} J_{L}^{a \mu}=\mathcal{O}_{L L}^{l}, \quad J_{Q \mu}^{a} J_{Q}^{a \mu}=4 \mathcal{O}_{L L}^{(8) q}+\frac{2-N_{c}}{N_{c}} \mathcal{O}_{L L}^{q},
\end{aligned}
$$

and

$$
g J_{W}^{a \mu} J_{F \mu}^{a}=g\left(\bar{F}_{L} \gamma^{\mu} \sigma^{a} F_{L}\right)\left(D^{\nu} W_{\mu \nu}^{a}\right) \equiv \mathcal{O}_{W L}^{F},
$$

while a heavy $S U(3)_{c}$-octet vector could give:

$$
J_{u \mu}^{A} J_{u}^{A \mu}=(1 / 3) \mathcal{O}_{R R}^{u}, \quad J_{Q \mu}^{A} J_{Q}^{A \mu}=\mathcal{O}_{L L}^{(8) q}, \quad J_{Q \mu}^{A} J_{u}^{A \mu}=\mathcal{O}_{L R}^{(8) u} .
$$

\section{$\circ$ Fermion $\times$ fermion}

Finally, we list operators that can arise from integrating a heavy fermion. If the fermion is a singlet:

$$
\begin{gathered}
\bar{J}_{y_{u} \bar{u}_{R}} i \not D J_{D u}=y_{u} D_{\mu}\left(\bar{Q}_{L} \widetilde{H}\right) \gamma^{\mu} \gamma^{\nu} D_{\nu} u_{R} \equiv \mathcal{O}_{y R}^{u}, \\
\bar{J}_{y_{u} \bar{u}_{R}} i \not D J_{y_{u} \bar{u}_{R}}+\text { h.c. }=\frac{1}{2}\left|y_{u}\right|^{2}\left[-\widetilde{\mathcal{O}}_{L}^{(3) q}+\widetilde{\mathcal{O}}_{L}^{q}-\mathcal{O}_{L}^{(3) q}+\mathcal{O}_{L}^{q}\right],
\end{gathered}
$$

where

$$
\begin{aligned}
\widetilde{\mathcal{O}}_{L}^{(3) q} & =i\left(\bar{Q}_{L} \sigma^{a} \stackrel{\leftrightarrow}{\not D} Q_{L}\right)\left(H^{\dagger} \sigma^{a} H\right) \\
\widetilde{\mathcal{O}}_{L}^{q} & =i\left(\bar{Q}_{L} \stackrel{\leftrightarrow}{\not D} Q_{L}\right)|H|^{2}
\end{aligned}
$$

are redundant operators.

If the fermion integrated-out is a doublet, one can get:

$$
\begin{aligned}
\bar{J}_{D Q} i \not D J_{y_{u} \bar{Q}_{L}} & =y_{u} D_{\mu} \bar{Q}_{L} \gamma^{\mu} \gamma^{\nu} D_{\nu}\left(\widetilde{H} u_{R}\right) \equiv \mathcal{O}_{y L}^{u}, \\
\bar{J}_{y_{u} \bar{Q}_{L}} i \not D J_{y_{u} \bar{Q}_{L}}+\text { h.c. } & =\left|y_{u}\right|^{2}\left[-\mathcal{O}_{R}^{u}+\widetilde{\mathcal{O}}_{R}^{u}\right]
\end{aligned}
$$

with the redundant operator:

$$
\widetilde{\mathcal{O}}_{R}^{u}=i\left(\bar{u}_{R} \stackrel{\leftrightarrow}{\not D} u_{R}\right)|H|^{2}
$$

Finally, from integrating out a heavy fermion triplet, we can get:

$$
\begin{aligned}
& \bar{J}_{\widetilde{H} F}^{a} i \not D J_{\widetilde{H} F}^{a}+\text { h.c. }=\frac{1}{2}\left[\widetilde{\mathcal{O}}_{L}^{(3) F}+3 \widetilde{\mathcal{O}}_{L}^{F}+\mathcal{O}_{L}^{(3) F}+3 \mathcal{O}_{L}^{F}\right], \\
& \bar{J}_{H F}^{a} i \not D J_{H F}^{a}+\text { h.c. }=\frac{1}{2}\left[-\widetilde{\mathcal{O}}_{L}^{(3) F}+3 \widetilde{\mathcal{O}}_{L}^{F}+\mathcal{O}_{L}^{(3) F}-3 \mathcal{O}_{L}^{F}\right] .
\end{aligned}
$$

To describe the effect of a heavy fermion that is a color octet, one would need to generalize the quark currents of Subsection A.1 by inserting $S U(3)_{c}$ generators. However, the dimension-6 operators that result have been already found in Eqs. (A.18) and (A.20). 


\section{A.3 Field redefinitions and redundant operators}

Many $d=6$ current-current operators are redundant: they can be removed from the Lagrangian by field redefinitions. We will show how field redefinitions can be used for that purpose, focusing here on current-current operators not of the 4-fermion type.

Let us start first with bosonic operators. Consider the following transformations that shift fields by some of the bosonic currents listed in Subsection A.1 (with the same quantum numbers of the shifted fields):

$$
\begin{aligned}
H \rightarrow H+\alpha_{1} J_{H}^{(2)} / \Lambda^{2}, & H \rightarrow H\left(1-\alpha_{2} m^{2} / \Lambda^{2}\right)+\alpha_{2} J_{\square H} / \Lambda^{2}, \\
B_{\mu} \rightarrow B_{\mu}+\left[g^{\prime} \alpha_{B} J_{H \mu}+\alpha_{2 B} J_{B \mu}\right] / \Lambda^{2}, & W_{\mu}^{a} \rightarrow W_{\mu}^{a}+\left[g \alpha_{W} J_{H \mu}^{a}+\alpha_{2 W} J_{W \mu}^{a}\right] / \Lambda^{2}, \\
G_{\mu}^{A} \rightarrow G_{\mu}^{A}+\alpha_{2 G} J_{G \mu}^{A} / \Lambda^{2}, &
\end{aligned}
$$

with $\alpha_{i}$ arbitrary parameters (taken real). These transformations induce shifts in the $d=6$ Wilson coefficients ${ }^{14}$ of Eqs. (4) and (5) plus the redundant operator $\mathcal{O}_{K 4}=\left|D_{\mu}^{2} H\right|^{2}$ :

$$
\begin{aligned}
& c_{H} \rightarrow c_{H}+2 \alpha_{1}+\left(4 \lambda \alpha_{2}-\alpha_{W} g^{2}\right) / g_{*}^{2}, \\
& c_{r} \rightarrow c_{r}+2 \alpha_{1}+\left(4 \lambda \alpha_{2}+2 \alpha_{W} g^{2}\right) / g_{*}^{2}, \\
& c_{6} \rightarrow c_{6}-4 \alpha_{1} \\
& c_{T} \rightarrow c_{T}-\alpha_{B} g^{\prime 2} / g_{*}^{2}, \\
& c_{B} \rightarrow c_{B}-2 \alpha_{B}+\alpha_{2 B}, \\
& c_{W} \rightarrow c_{W}-2 \alpha_{W}+\alpha_{2 W} \\
& c_{2 W} \rightarrow c_{2 W}+2 \alpha_{2 W} \\
& c_{2 B} \rightarrow c_{2 B}+2 \alpha_{2 B} \\
& c_{2 G} \rightarrow c_{2 G}+2 \alpha_{2 G} \\
& c_{K 4} \rightarrow c_{K 4}-2 \alpha_{2} .
\end{aligned}
$$

Notice that only operators of tree-level type are shifted. Using this shift freedom, we could eliminate 7 out of the 10 operators $\left\{\mathcal{O}_{H}, \mathcal{O}_{r}, \mathcal{O}_{6}, \mathcal{O}_{T}, \mathcal{O}_{B}, \mathcal{O}_{W}, \mathcal{O}_{2 W}, \mathcal{O}_{2 B}, \mathcal{O}_{K 4}, \mathcal{O}_{2 G}\right\}$ by choosing appropriately the $\alpha_{i}$ 's and leave only $\mathcal{O}_{H}, \mathcal{O}_{T}$ and $\mathcal{O}_{6}$. As we discussed in Section 2, however, it is convenient to keep the operators $\mathcal{O}_{W}$ and $\mathcal{O}_{B}$ in the basis, in which we could also keep $\mathcal{O}_{2 W}, \mathcal{O}_{2 B}$ and $\mathcal{O}_{2 G}$. If we do not use 5 of these shifts to remove $\mathcal{O}_{W}, \mathcal{O}_{B}, \mathcal{O}_{2 W}$, $\mathcal{O}_{2 B}$ and $\mathcal{O}_{2 G}$, they can be used later on to remove 5 other operators involving fermions. We will discuss such operators next.

Besides the bosonic redundant operators discussed above, there are redundant operators that involve Higgs and fermion fields. For instance, we have the following first-class operators:

$$
\widetilde{\mathcal{O}}_{L}^{F}=\left(i \bar{F}_{L} \stackrel{\leftrightarrow}{\not D} F_{L}\right)|H|^{2}, \quad \widetilde{\mathcal{O}}_{L}^{(3) F}=\left(i \bar{F}_{L} \sigma^{a} \stackrel{\leftrightarrow}{\not D} F_{L}\right)\left(H^{\dagger} \sigma^{a} H\right), \quad \widetilde{\mathcal{O}}_{R}^{f}=\left(i \bar{f}_{R} \stackrel{\leftrightarrow}{\not D} f_{R}\right)|H|^{2}
$$

\footnotetext{
${ }^{14}$ Shifts of order $m^{2} / \Lambda^{2}$ induced on the renormalizable dimension-4 SM operators play no role. There are also shifts in the coefficients of the operators made of fermions that we show below.
} 
as well as the second-class operators

$$
\begin{array}{cl}
\mathcal{O}_{y H}^{u}=y_{u} D_{\mu}\left(\bar{Q}_{L} u_{R}\right) D^{\mu} \widetilde{H}, & \mathcal{O}_{y R}^{u}=y_{u} D_{\mu}\left(\bar{Q}_{L} \widetilde{H}\right) \gamma^{\mu} \gamma^{\nu} D_{\nu} u_{R}, \\
\mathcal{O}_{y L}^{u}=y_{u} D_{\mu} \bar{Q}_{L} \gamma^{\mu} \gamma^{\nu} D_{\nu}\left(\widetilde{H} u_{R}\right), & \mathcal{O}_{y L R}^{u}=y_{u}\left(D_{\mu} \bar{Q}_{L}\right) \gamma^{\mu} \gamma^{\nu}\left(D_{\nu} u_{R}\right) \tilde{H}
\end{array}
$$

(and similar operators for down-type quarks and leptons). In addition, there are (second-class) operators involving fermions and gauge bosons:

$$
\begin{aligned}
& \mathcal{O}_{B L}^{F}=g^{\prime}\left(\bar{F}_{L} \gamma^{\mu} F_{L}\right) \partial^{\nu} B_{\mu \nu}, \quad \mathcal{O}_{B R}^{f}=g^{\prime}\left(\bar{f}_{R} \gamma^{\mu} f_{R}\right) \partial^{\nu} B_{\mu \nu}, \quad \mathcal{O}_{W L}^{F}=g\left(\bar{F}_{L} \sigma^{a} \gamma^{\mu} F_{L}\right) D^{\nu} W_{\mu \nu}^{a}, \\
& \mathcal{O}_{G L}^{q}=g_{s}\left(\bar{Q}_{L} T^{A} \gamma^{\mu} Q_{L}\right) D^{\nu} G_{\mu \nu}^{A}, \quad \mathcal{O}_{G R}^{f}=g_{s}\left(\bar{f}_{R} T^{A} \gamma^{\mu} f_{R}\right) D^{\nu} G_{\mu \nu}^{A} .
\end{aligned}
$$

To see that the operators (A.25)-(A.27) can indeed be removed from the Lagrangian, consider the following field redefinitions that involve fermions:

$$
\begin{aligned}
Q_{L} \rightarrow & Q_{L}\left(1+g_{*}^{2} \alpha_{L} J_{H} / \Lambda^{2}\right)+\left[g_{*}^{2} \alpha_{L}^{(3)} J_{H}^{a} \sigma^{a} Q_{L}+i \alpha_{y L}^{u} \not D J_{y_{u} \bar{Q}_{L}}+y_{u} \alpha_{y L R}^{u} \widetilde{H} J_{D u_{R}}\right. \\
& \left.+i \alpha_{y L}^{d} \not D J_{y_{d} \bar{Q}_{L}}+y_{d} \alpha_{y L R}^{d} H J_{D d_{R}}\right] / \Lambda^{2} \\
u_{R} \rightarrow & u_{R}\left(1+g_{*}^{2} \alpha_{R}^{u} J_{H} / \Lambda^{2}\right)+i \alpha_{y R}^{u} \not D J_{y_{u} \bar{u}_{R}} / \Lambda^{2} \\
d_{R} \rightarrow & d_{R}\left(1+g_{*}^{2} \alpha_{R}^{d} J_{H} / \Lambda^{2}\right)+i \alpha_{y R}^{d} \not D J_{y_{d} \bar{d}_{R}} / \Lambda^{2} \\
B_{\mu} \rightarrow & B_{\mu}+g^{\prime}\left[\alpha_{F_{L}}^{B} J_{F \mu}+\alpha_{f_{R}}^{B} J_{f f \mu}\right] / \Lambda^{2} \\
W_{\mu}^{a} \rightarrow & W_{\mu}^{a}+g \alpha_{f_{L}}^{W} J_{L \mu}^{a} / \Lambda^{2} \\
G_{\mu}^{A} \rightarrow & G_{\mu}^{A}+g_{s}\left[\alpha_{F_{L}}^{G} J_{F \mu}^{A}+\alpha_{f_{R}}^{G} J_{f \mu}^{A}\right] / \Lambda^{2} \\
\widetilde{H} \rightarrow & \widetilde{H}+\alpha_{H t} J_{y_{t} H}^{\dagger} / \Lambda^{2} \\
H \rightarrow & H+\alpha_{H b} J_{y_{b} H}^{\dagger} / \Lambda^{2}
\end{aligned}
$$

under which the Wilson coefficients shift as follows: For the Higgs-fermion operators of Eq. (14), plus the straightforward generalization to the down-type fermions, and the up-down mixed operator of Eq. (17), we get (for third-generation quarks):

$$
\begin{aligned}
c_{y_{t}} & \rightarrow c_{y_{t}}-\alpha_{1}-2 \frac{\lambda}{g_{*}^{2}} \alpha_{H t}-\alpha_{R}^{t}-\alpha_{L}+\alpha_{L}^{(3)}, \\
c_{y_{b}} & \rightarrow c_{y_{b}}-\alpha_{1}-2 \frac{\lambda}{g_{*}^{2}} \alpha_{H b}-\alpha_{R}^{b}-\alpha_{L}-\alpha_{L}^{(3)}, \\
c_{R} & \rightarrow c_{R}+\frac{\left|y_{t}\right|^{2}}{g_{*}^{2}} \alpha_{y L}^{t}+\frac{g^{\prime 2}}{2 g_{*}^{2}}\left(2 Y_{R}^{t} \alpha_{B}+\alpha_{t_{R}}^{B}\right), \\
c_{L}^{q_{3}} & \rightarrow c_{L}^{q_{3}}-\frac{1}{2} \frac{\left|y_{t}\right|^{2}}{g_{*}^{2}} \alpha_{y R}^{t}+\frac{g^{\prime 2}}{2 g_{*}^{2}}\left(2 Y_{L}^{q} \alpha_{B}+\alpha_{Q_{L}}^{B}\right), \\
c_{L}^{(3) q_{3}} & \rightarrow c_{L}^{(3) q_{3}}+\frac{1}{2} \frac{\left|y_{t}\right|^{2}}{g_{*}^{2}} \alpha_{y R}^{t}+\frac{g^{2}}{2 g_{*}^{2}}\left(\alpha_{W}+\alpha_{Q_{L}}^{W}\right), \\
c_{R}^{t b} & \rightarrow c_{R}^{t b}-\frac{1}{2}\left(\alpha_{y L}^{t}+\alpha_{y L}^{b}\right) .
\end{aligned}
$$


The Higgs-fermion redundant operators of Eq. (A.25) can be eliminated by the shifts:

$$
\begin{aligned}
\tilde{c}_{R}^{t} & \rightarrow \tilde{c}_{R}^{t}+\alpha_{R}^{t}-\frac{\left|y_{t}\right|^{2}}{g_{*}^{2}}\left(\alpha_{y L}^{t}+\alpha_{y L R}^{t}\right), \\
\tilde{c}_{R}^{b} & \rightarrow \tilde{c}_{R}^{b}+\alpha_{R}^{b}, \\
\tilde{c}_{L}^{q_{3}} & \rightarrow \tilde{c}_{L}^{q_{3}}+\alpha_{L}-\frac{1}{2} \frac{\left|y_{t}\right|^{2}}{g_{*}^{2}} \alpha_{y R}^{t} \\
\tilde{c}_{L}^{(3) q_{3}} & \rightarrow \tilde{c}_{L}^{(3) q_{3}}+\alpha_{L}^{(3)}+\frac{1}{2} \frac{\left|y_{t}\right|^{2}}{g_{*}^{2}} \alpha_{y R}^{t}
\end{aligned}
$$

(where, from here on, we neglect $\left|y_{b}\right|^{2}$ and $\left|y_{\tau}\right|^{2}$ contributions) while the redundant Higgsfermion operators of Eq. (A.26) can be eliminated by the shifts:

$$
\begin{aligned}
c_{y H}^{f} & \rightarrow c_{y H}^{f}+\alpha_{H f}+\alpha_{2}, \\
c_{y R}^{f} & \rightarrow c_{y R}^{f}+\alpha_{y R}^{f}, \\
c_{y L}^{f} & \rightarrow c_{y L}^{f}+\alpha_{y L}^{f}, \\
c_{y L R}^{f} & \rightarrow c_{y L R}^{f}+\alpha_{y L R}^{f} .
\end{aligned}
$$

All the redundant gauge-fermion operators of Eq. (A.27) can be removed by the shifts:

$$
\begin{aligned}
c_{B R}^{f} & \rightarrow c_{B R}^{f}+Y_{R}^{f} \alpha_{2 B}-\alpha_{f_{R}}^{B}, \\
c_{B L}^{F} & \rightarrow c_{B L}^{F}+Y_{L}^{F} \alpha_{2 B}-\alpha_{F_{L}}^{B}, \\
c_{W L}^{F} & \rightarrow c_{W L}^{F}+\frac{1}{2} \alpha_{2 W}-\alpha_{F_{L}}^{W}, \\
c_{G L, G R}^{q} & \rightarrow c_{G L, G R}^{q}+\alpha_{2 G}-\alpha_{q_{L, R}}^{G} .
\end{aligned}
$$

Finally, the coefficients of four-fermion operators will also be shifted but we will not need such shifts and we do not list them.

Using all the shift freedom to remove these redundant operators we end up (say, for the third family) with the following Higgs-fermion Wilson coefficients: $y_{f} c_{y_{f}}, c_{R}^{f}, c_{L}^{F}, c_{L}^{(3) F}$ and $c_{R}^{t b}$, with $f=t, b, \tau$ and $F=q, l$, in agreement with the operators listed in Table 2 of Section 2. 


\section{B Anomalous dimensions of $d=6$ Wilson coefficients}

In our analysis we are interested in potentially large radiative effects in the running of the $d=6$ Wilson coefficients $c_{i}$ from the scale $\Lambda$ of new physics to the electroweak scale. To study such effects we have computed the one-loop anomalous dimensions $\gamma_{c_{i}}$ for the Wilson coefficients, which are functions of the coefficients themselves, that is:

$$
\gamma_{c_{i}}=\frac{d c_{i}}{d \log \mu}=\gamma_{c_{i}}\left(c_{j}\right)
$$

where $\mu$ is the renormalization scale.

When redundant operators are removed from the Lagrangian some care has to be taken in computing anomalous dimensions of the operators left in the basis. The reason is that redundant operators can be generated through $\mathrm{RG}$ evolution by operator mixing with nonredundant operator. In other words, the $\gamma_{c_{i}}$ 's of redundant operators are not zero in general.

Let us explain how this effect can be taken care of in a simple way. Consider a basis formed by a set of coefficients $\left\{c_{i}\right\}$, after removing a set of redundant coefficients $\left\{c_{i}^{r}\right\}$. The procedure to remove the $c_{i}^{r}$ is straightforward and has been illustrated in the previous Appendix. One starts from the shifts induced by field-redefinitions with arbitrary parameters $\alpha_{k}$, which have the form

$$
c_{i} \rightarrow c_{i}^{\prime}\left(\alpha_{j}\right)=c_{i}+\sum_{k} s_{i k} \alpha_{k}, \quad c_{i}^{r} \rightarrow c_{i}^{r \prime}\left(\alpha_{j}\right)=c_{i}^{r}+\sum_{k} s_{i k}^{r} \alpha_{k} .
$$

Then the $\alpha_{k}$ 's are chosen so as to remove the redundant operators,

$$
c_{i}^{r \prime}\left(\alpha_{j}^{*}\right)=0 \Rightarrow \alpha_{j}^{*}=-\sum_{i}\left(s^{r}\right)_{j i}^{-1} c_{i}^{r} .
$$

It is then convenient to define the following combinations

$$
C_{i} \equiv c_{i}^{\prime}\left(\alpha_{j}^{*}\right)=c_{i}-\sum_{k l} s_{i k}\left(s^{r}\right)_{k l}^{-1} c_{l}^{r}
$$

which are invariant under the arbitrary shifts (B.2) and correspond to a more physical definition of the Wilson coefficients.

The anomalous dimensions of these shift-invariant $C_{i}$ 's are simply

$$
\gamma_{C_{i}}=\gamma_{c_{i}}-\sum_{k l} s_{i k}\left(s^{r}\right)_{k l}^{-1} \gamma_{c_{l}^{r}}=\gamma_{i}\left(c_{k} ; c_{k}^{r}\right)
$$

where the last expression just indicates some function of the Wilson coefficients and we distinguish in its argument between coefficients in the basis and coefficients of redundant operators. The key property of this function is that it must depend on the Wilson coefficients only through the shift-invariant combinations. That is, it satisfies

$$
\gamma_{i}\left(c_{k} ; c_{k}^{r}\right)=\gamma_{i}\left(C_{k} ; 0\right)
$$


This implies that setting $c_{k}^{r}=0$ in these $\gamma_{i}\left(c_{k} ; c_{k}^{r}\right)$ functions is now a consistent procedure to obtain the anomalous dimensions after removing redundant operators. ${ }^{15}$ An explicit example of this is given at the end of Appendix B. We applied this procedure to calculate the anomalous dimensions used in Section 5. In the next Subsections we will list the required shift-invariant $C_{i}$ combinations and present the $\gamma_{c_{i}}$ 's necessary to complete the calculation.

\section{B.1 Shift-invariant combinations of Wilson coefficients}

In order to simplify the expressions for the shift-invariant combinations $C_{i}$ of Wilson coefficients, we present them first in a basis that treats as redundant the operators $\mathcal{O}_{B}, \mathcal{O}_{W}, \mathcal{O}_{2 B}$, $\mathcal{O}_{2 W}$ and $\mathcal{O}_{2 G}$. We explain afterwards how to express these combinations in other bases, as those that keep these operators. As we are not interested in this paper in calculating the anomalous dimensions of 4 -fermion operators, below we restrict our $C_{i}$ 's to non-4-fermion current-current operators. We find:

$$
\begin{aligned}
& C_{H} \equiv c_{H}-c_{r}-\frac{3 g^{2}}{4 g_{*}^{2}}\left(2 c_{W}-c_{2 W}\right) \\
& C_{T} \equiv c_{T}-\frac{g^{\prime 2}}{4 g_{*}^{2}}\left(2 c_{B}-c_{2 B}\right) \\
& C_{6} \equiv c_{6}+2 c_{r}+\frac{g^{2}}{g_{*}^{2}}\left(2 c_{W}-c_{2 W}\right)+4 \frac{\lambda}{g_{*}^{2}} c_{K 4}, \\
& C_{y_{t}}=c_{y_{t}}+\frac{1}{2} c_{r}+2 \frac{\lambda}{g_{*}^{2}}\left(c_{y H}^{t}+c_{K 4}\right)+\tilde{c}_{R}^{t}+\tilde{c}_{L}^{q_{3}}-\tilde{c}_{L}^{(3) q_{3}}+\frac{g^{2}}{4 g_{*}^{2}}\left(2 c_{W}-c_{2 W}\right) \\
& +\frac{\left|y_{t}\right|^{2}}{g_{*}^{2}}\left(c_{y R}^{t}+c_{y L}^{t}+c_{y L R}^{t}\right) \\
& C_{y_{b}}=c_{y_{b}}+\frac{1}{2} c_{r}+2 \frac{\lambda}{g_{*}^{2}}\left(c_{y H}^{b}+c_{K 4}\right)+\tilde{c}_{R}^{b}+\tilde{c}_{L}^{q_{3}}+\tilde{c}_{L}^{(3) q_{3}}+\frac{g^{2}}{4 g_{*}^{2}}\left(2 c_{W}-c_{2 W}\right), \\
& C_{y_{\tau}}=c_{y_{\tau}}+\frac{1}{2} c_{r}+2 \frac{\lambda}{g_{*}^{2}}\left(c_{y H}^{\tau}+c_{K 4}\right)+\tilde{c}_{R}^{\tau}+\tilde{c}_{L}^{l_{3}}+\tilde{c}_{L}^{(3) l_{3}}+\frac{g^{2}}{4 g_{*}^{2}}\left(2 c_{W}-c_{2 W}\right), \\
& C_{R}^{t}=c_{R}^{t}-\frac{\left|y_{t}\right|^{2}}{g_{*}^{2}} c_{y L}^{t}+\frac{g^{\prime 2}}{2 g_{*}^{2}}\left[Y_{R}^{t}\left(c_{B}-c_{2 B}\right)+c_{B R}^{t}\right] \\
& C_{R}^{b}=c_{R}^{b}+\frac{g^{\prime 2}}{2 g_{*}^{2}}\left[Y_{R}^{b}\left(c_{B}-c_{2 B}\right)+c_{B R}^{b}\right], \\
& C_{R}^{\tau}=c_{R}^{\tau}+\frac{g^{\prime 2}}{2 g_{*}^{2}}\left[Y_{R}^{\tau}\left(c_{B}-c_{2 B}\right)+c_{B R}^{\tau}\right], \\
& C_{L}^{q_{3}}=c_{L}^{q_{3}}+\frac{1}{2} \frac{\left|y_{t}\right|^{2}}{g_{*}^{2}} c_{y R}^{t}+\frac{g^{\prime 2}}{2 g_{*}^{2}}\left[Y_{L}^{q}\left(c_{B}-c_{2 B}\right)+c_{B L}^{q_{3}}\right], \\
& C_{L}^{l_{3}}=c_{L}^{l_{3}}+\frac{g^{2}}{2 g_{*}^{2}}\left[Y_{L}^{l}\left(c_{B}-c_{2 B}\right)+c_{B L}^{l_{3}}\right]
\end{aligned}
$$

\footnotetext{
${ }^{15}$ This is equivalent to the procedure described independently in [32].
} 


$$
\begin{aligned}
C_{L}^{(3) q_{3}} & =c_{L}^{(3) q_{3}}-\frac{1}{2} \frac{\left|y_{t}\right|^{2}}{g_{*}^{2}} c_{y R}^{t}+\frac{g^{2}}{4 g_{*}^{2}}\left(c_{W}-c_{2 W}+2 c_{W L}^{q_{3}}\right) \\
C_{L}^{(3) l_{3}} & =c_{L}^{(3) l_{3}}+\frac{g^{2}}{4 g_{*}^{2}}\left(c_{W}-c_{2 W}+2 c_{W L}^{l_{3}}\right) \\
C_{R}^{t b} & =c_{R}^{t b}+\frac{1}{2}\left(c_{y L}^{t}+c_{y L}^{b}\right) .
\end{aligned}
$$

Out of the 59 independent operators for a single family, 20 are of one-loop type and 25 are 4 -fermion tree-level operators. The remaining 14 are tree-level operators whose number corresponds to the 14 physical $C_{i}$ 's in (B.7).

Let us now discuss how these $C_{i}$ 's would be modified in other basis. For example, if we keep $\mathcal{O}_{B}$ and $\mathcal{O}_{W}$ in the basis instead of the leptonic operators $\mathcal{O}_{L}^{l}$ and $\mathcal{O}_{L}^{(3) l}$, then one should remove $C_{L}^{l}$ and $C_{L}^{(3) l}$ from the list of $C_{i}$ 's. This is accomplished by making the replacements

$$
\begin{gathered}
c_{B} \rightarrow c_{2 B}-\frac{1}{Y_{L}^{l}} c_{B L}^{l}-\frac{2 g_{*}^{2}}{Y_{L}^{l} g^{\prime 2}} c_{L}^{l}, \\
c_{W} \rightarrow c_{2 W}-2 c_{W L}^{l}-4 \frac{g_{*}^{2}}{g^{2}} c_{L}^{(3) l},
\end{gathered}
$$

in all the $C_{i}$ 's (obtaining in particular $C_{L}^{l}=C_{L}^{(3) l}=0$ ) and then add to the list the following two new $C_{i}$ 's:

$$
\begin{aligned}
C_{B} & =c_{B}-c_{2 B}+\frac{1}{Y_{L}^{l}} c_{B L}^{l}+\frac{2 g_{*}^{2}}{Y_{L}^{l} g^{\prime 2}} c_{L}^{l}, \\
C_{W} & =c_{W}-c_{2 W}+2 c_{W L}^{l}+4 \frac{g_{*}^{2}}{g^{2}} c_{L}^{(3) l} .
\end{aligned}
$$

The replacement in Eq. (B.8) introduces a dependence on $\gamma_{c_{B L}^{l}}, \gamma_{c_{c_{L}}^{l}}, \gamma_{c_{W L}^{l}}$ and $\gamma_{c_{L}^{(3) l}}$ in the calculation of the anomalous dimensions of the $C_{i}$ 's, but the only non-redundant coefficients that appear in those anomalous dimensions depend on leptonic Yukawa couplings that we neglect.

In a similar way, $\mathcal{O}_{2 B}, \mathcal{O}_{2 W}$ and $\mathcal{O}_{2 G}$ can be kept in the basis instead of three 4-fermion operators of the first family, e.g. $\mathcal{O}_{R R}^{e}, \mathcal{O}_{L L}^{l}$ and $\mathcal{O}_{R R}^{(8) d}$. In this basis $c_{2 B}, c_{2 W}$ and $c_{2 G}$ have to be replaced by linear combinations of $c_{R R}^{e}, c_{L L}^{l}$ and $c_{R R}^{(8) d}$ in the $C_{i}$ 's above. However, this replacement has no impact on the anomalous dimensions of the $C_{i}$ 's if we only keep the coefficients of Eq. (53) and neglect small Yukawas. Indeed, it is simple to realize that Eq. (53) can only renormalize $c_{2 B, 2 W, 2 G}$ or $c_{R R}^{e}, c_{L L}^{l}$ and $c_{R R}^{(8) d}$ through lepton or down Yukawas, which are terms we neglect in our RGEs. Therefore, whether we keep $\mathcal{O}_{2 B, 2 W, 2 G}$ or 4-fermion operators, the RGEs given in the main body of the paper are unaffected.

\section{B.2 Anomalous dimensions before removing redundant operators}

To calculate the anomalous dimensions $\gamma_{C_{i}}$ 's, following Eq. (B.5), we need to calculate the anomalous dimensions of the Wilson coefficients entering in the $C_{i}$ 's, including those that are redundant. 
We have calculated these anomalous dimensions to linear order in the $c_{j}$ 's of Eq. (53), the only exception being $c_{r}$, which we keep for illustrative purposes here. Parametrically one has $\gamma_{c_{i}} \sim g_{j}^{2} c_{j} / 16 \pi^{2}$ and we only keep $g_{j}^{2}=\left\{y_{t}^{2}, g_{s}^{2}, g^{2}, g^{\prime 2}, \lambda\right\}$, dropping $g_{j}^{2}=\left\{y_{b}^{2}, y_{\tau}^{2}, \ldots\right\}$. The anomalous dimensions, calculated in Landau gauge, are:

$$
\begin{aligned}
& \gamma_{c_{H}}=\frac{1}{4 \pi^{2}}\left\{N_{c} y_{t}^{2}\left[c_{y_{t}}+c_{L}^{(3)}\right]+\lambda\left(7 c_{H}-c_{r}\right)+\frac{3}{8}\left[g^{2}\left(c_{H}+2 c_{r}\right)+g^{\prime 2} c_{r}\right]\right\}-4 \gamma_{h} c_{H}, \\
& \gamma_{c_{T}}=\frac{1}{16 \pi^{2}}\left[4 N_{c} y_{t}^{2}\left(c_{R}-c_{L}\right)+\frac{3}{2} g^{\prime 2}\left(c_{H}-c_{r}\right)\right]-4 \gamma_{h} c_{T} \\
& \gamma_{\lambda c_{6}}=\frac{1}{8 \pi^{2}}\left\{54 \lambda^{2} c_{6}-4 N_{c} y_{t}^{4} c_{y_{t}}+12 \lambda^{2}\left(3 c_{H}+2 c_{r}\right)-\frac{3}{8}\left[2 g^{4}+\left(g^{2}+g^{\prime 2}\right)^{2}\right] c_{r}\right\}-6 \gamma_{h} \lambda c_{6} \text {, } \\
& \frac{1}{y_{t}} \gamma_{y_{t} c_{y_{t}}}=\frac{1}{16 \pi^{2}}\left\{4 \lambda\left[c_{R}-c_{L}+3 c_{L}^{(3)}+6 c_{y_{t}}\right]-g^{\prime 2}\left[c_{R}+4 c_{L}-4 c_{L}^{(3)}+\frac{2}{3} c_{y_{t}}\right]\right. \\
& \left.-3 g^{2} c_{R}-8 g_{s}^{2} c_{y_{t}}+2 y_{t}^{2}\left[4 c_{L R}+4 C_{F} c_{L R}^{(8)}+c_{R}-c_{L}+c_{L}^{(3)}+2 c_{y_{t}}+c_{H}\right]\right\} \\
& -\left(3 \gamma_{h}+\gamma_{Q_{L}}+\gamma_{t_{R}}\right) c_{y_{t}}, \\
& \frac{1}{y_{b}} \gamma_{y_{b} c_{y_{b}}}=\frac{1}{8 \pi^{2}}\left\{2 \lambda\left[c_{L}+3 c_{L}^{(3)}+6 c_{y_{b}}\right]+y_{t}^{2}\left[2 c_{L}^{(3)}-c_{y_{t}}\right]+\left(\frac{1}{6} g^{\prime 2}-4 g_{s}^{2}\right) c_{y_{b}}\right. \\
& \left.+\frac{y_{t}^{2}}{g_{*}^{2}}\left[3 g^{2}-2 y_{t}^{2}-4 \lambda\right] c_{R}^{t b}+g^{\prime 2}\left[c_{L}+c_{L}^{(3)}\right]-\frac{y_{t}^{4}}{g_{*}^{2}}\left[\left(2 N_{c}+1\right) c_{y_{t} y_{b}}+C_{F} c_{y_{t} y_{b}}^{(8)}\right]\right\} \\
& -\left(\gamma_{Q_{L}}+\gamma_{b_{R}}+3 \gamma_{h}\right) c_{y_{b}} \text {, } \\
& \frac{1}{y_{\tau}} \gamma_{y_{\tau} c_{y_{\tau}}}=\frac{1}{16 \pi^{2}}\left[3\left(8 \lambda-g^{\prime 2}\right) c_{y_{\tau}}+2 N_{c} \frac{y_{t}^{2}}{g_{*}^{2}}\left(\lambda-y_{t}^{2}\right)\left(2 c_{y_{t} y_{\tau}}+c_{y_{t} y_{\tau}}^{\prime}\right)\right] \\
& -\left(\gamma_{L_{L}}+\gamma_{\tau_{R}}+3 \gamma_{h}\right) c_{y_{\tau}} \\
& \gamma_{c_{R}}=\frac{1}{8 \pi^{2}}\left\{y_{t}^{2}\left[N_{c} c_{L R}-2\left(N_{c}+1\right) c_{R R}+2 c_{R}-c_{L}+\frac{1}{4}\left(c_{H}-c_{r}\right)\right]-\frac{3}{4}\left(3 g^{2}+g^{\prime 2}\right) c_{R}\right\} \\
& -2\left(\gamma_{h}+\gamma_{t_{R}}\right) c_{R} \\
& \gamma_{c_{L}}=\frac{1}{8 \pi^{2}}\left\{y_{t}^{2}\left[-N_{c} c_{L R}-\frac{1}{2} c_{R}+c_{L}-3 c_{L}^{(3)}-\frac{1}{8}\left(c_{H}-c_{r}\right)+\left(2 N_{c}+1\right) c_{L L}+C_{F} c_{L L}^{(8)}\right]\right. \\
& \left.-\frac{3}{4}\left(3 g^{2}+g^{\prime 2}\right) c_{L}\right\}-2\left(\gamma_{h}+\gamma_{Q_{L}}\right) c_{L} \\
& \gamma_{c_{L}^{(3)}}=\frac{-1}{8 \pi^{2}}\left\{y_{t}^{2}\left[c_{L}+c_{L}^{(3)}-\frac{1}{8}\left(c_{H}-c_{r}\right)+c_{L L}+C_{F} c_{L L}^{(8)}\right]+\frac{3}{4}\left(g^{2}+g^{\prime 2}\right) c_{L}^{(3)}\right\} \\
& -2\left(\gamma_{h}+\gamma_{Q_{L}}\right) c_{L}^{(3)} \\
& \gamma_{c_{r}}=\frac{1}{4 \pi^{2}}\left\{N_{c} y_{t}^{2}\left[c_{y_{t}}-2 c_{L}^{(3)}\right]+\lambda\left(c_{H}+5 c_{r}\right)+\frac{3}{8}\left[\left(5 g^{2}+g^{\prime 2}\right) c_{r}-2 g^{2} c_{H}\right]\right\}-4 \gamma_{h} c_{r} \text {, }
\end{aligned}
$$




$$
\begin{aligned}
& \frac{1}{y_{t}} \gamma_{y_{t} c_{y H}}=-\frac{g_{*}^{2}}{4 \pi^{2}}\left[c_{L R}+C_{F} c_{L R}^{(8)}\right]-\left(\gamma_{h}+\gamma_{Q_{L}}+\gamma_{t_{R}}\right) c_{y H} \\
& \frac{1}{y_{t}} \gamma_{y_{t} c_{y R}}=\frac{g_{*}^{2}}{8 \pi^{2}}\left[c_{L}-3 c_{L}^{(3)}\right]-\left(\gamma_{h}+\gamma_{Q_{L}}+\gamma_{t_{R}}\right) c_{y R}, \\
& \frac{1}{y_{t}} \gamma_{y_{t} c_{y L}}=-\frac{g_{*}^{2}}{8 \pi^{2}} c_{R}-\left(\gamma_{h}+\gamma_{Q_{L}}+\gamma_{t_{R}}\right) c_{y L} \\
& \frac{1}{y_{t}} \gamma_{y_{t} c_{y L R}}=\frac{g_{*}^{2}}{16 \pi^{2}}\left[c_{R}-c_{L}+3 c_{L}^{(3)}\right]-\left(\gamma_{h}+\gamma_{Q_{L}}+\gamma_{t_{R}}\right) c_{y L R}, \\
& \frac{1}{y_{b}} \gamma_{y_{b} c_{y H}^{b}}=\frac{1}{16 \pi^{2}} y_{t}^{2}\left[\left(2 N_{c}+1\right) c_{y_{t} y_{b}}+C_{F} c_{y_{t} y_{b}}^{(8)}\right]-\left(\gamma_{h}+\gamma_{Q_{L}}+\gamma_{b_{R}}\right) c_{y H}^{b} \\
& \gamma_{c_{K 4}}=\frac{y_{t}^{2}}{4 \pi^{2}} N_{c} c_{y H}-2 \gamma_{h} c_{K 4} \\
& \gamma_{\tilde{c}_{R}}=\frac{y_{t}^{2}}{16 \pi^{2}}\left[3 c_{y_{t}}+c_{R}+\frac{1}{2}\left(c_{H}+2 c_{r}\right)\right]-2\left(\gamma_{h}+\gamma_{t_{R}}\right) \tilde{c}_{R}, \\
& \gamma_{\tilde{c}_{L}}=\frac{y_{t}^{2}}{32 \pi^{2}}\left[3 c_{y_{t}}-c_{L}+3 c_{L}^{(3)}+\frac{1}{2}\left(c_{H}+2 c_{r}\right)\right]-2\left(\gamma_{h}+\gamma_{Q_{L}}\right) \tilde{c}_{L}, \\
& \gamma_{\tilde{c}_{L}^{(3)}}=\frac{y_{t}^{2}}{32 \pi^{2}}\left[-c_{y_{t}}+c_{L}+c_{L}^{(3)}-\frac{1}{2} c_{H}\right]-2\left(\gamma_{h}+\gamma_{Q_{L}}\right) \tilde{c}_{L}^{(3)} \\
& \gamma_{c_{W}}=\frac{g_{*}^{2}}{48 \pi^{2}}\left[16 N_{c} c_{L}^{(3)}-\left(c_{H}+c_{T}\right)\right]-\left(2 \gamma_{h}+\gamma_{W}+\frac{1}{g} \beta_{g}\right) c_{W} \\
& \gamma_{c_{B}}=\frac{g_{*}^{2}}{48 \pi^{2}}\left[\frac{8 N_{c}}{3}\left(2 c_{R}+c_{L}\right)-\left(c_{H}+5 c_{T}\right)\right]-2 \gamma_{h} c_{B}, \\
& \gamma_{c_{B R}}=\frac{g_{*}^{2}}{12 \pi^{2}}\left\{\frac{1}{3}\left[4\left(N_{c}+1\right) c_{R R}+N_{c} c_{L R}\right]+c_{R}\right\}-2 \gamma_{t_{R}} c_{B R}, \\
& \gamma_{c_{B L}}=\frac{g_{*}^{2}}{12 \pi^{2}}\left\{\frac{1}{3}\left[\left(2 N_{c}+1\right) c_{L L}+C_{F} c_{L L}^{(8)}+N_{c} c_{L R}\right]+2 c_{L}\right\}-2 \gamma_{Q_{L}} c_{B L} \\
& \gamma_{c_{W L}}=\frac{g_{*}^{2}}{12 \pi^{2}}\left[c_{L L}+C_{F} c_{L L}^{(8)}+c_{L}^{(3)}\right]-\left(2 \gamma_{Q_{L}}+\gamma_{W}+\frac{1}{g} \beta_{g}\right) c_{W L},
\end{aligned}
$$

where $C_{F}=\left(N_{c}^{2}-1\right) /\left(2 N_{c}\right), \beta_{g}=\frac{d g}{d \log \mu}$ and

$$
\begin{aligned}
& \gamma_{h}=\frac{1}{16 \pi^{2}}\left[-N_{c} y_{t}^{2}+\frac{3}{4}\left(3 g^{2}+g^{\prime 2}\right)\right], \quad \gamma_{Q_{L}}=\frac{1}{16 \pi^{2}}\left[-\frac{1}{2} y_{t}^{2}\right], \quad \gamma_{t_{R}}=-\frac{y_{t}^{2}}{16 \pi^{2}} \\
& \gamma_{W}=-\frac{1}{g} \beta_{g}-\frac{3}{16 \pi^{2}} g^{2}=\frac{1}{16 \pi^{2}} \frac{g^{2}}{6}
\end{aligned}
$$

are the wave-function renormalization terms. The corresponding wave-function terms for leptons and $b_{R}\left(\gamma_{L_{L}}, \gamma_{\tau_{R}}\right.$ and $\left.\gamma_{b_{R}}\right)$ are proportional to small Yukawa couplings squared that we 


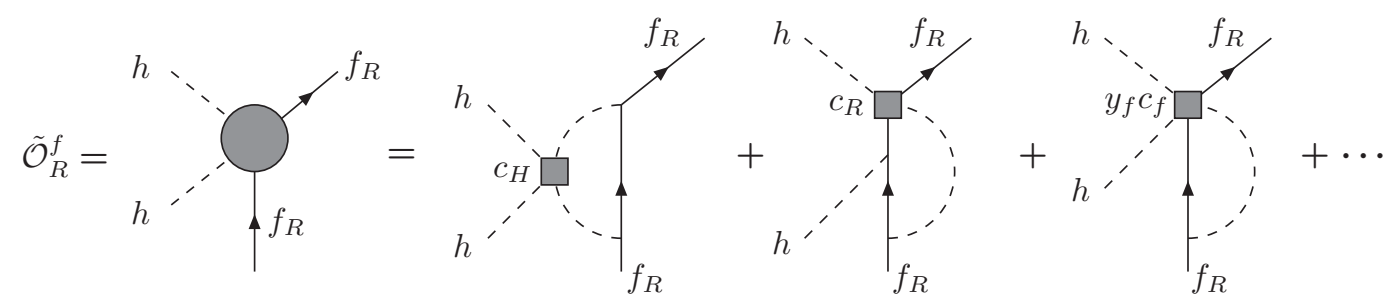

Figure 3: Diagrams that generate at one-loop the redundant operator $\tilde{\mathcal{O}}_{R}^{f}$.
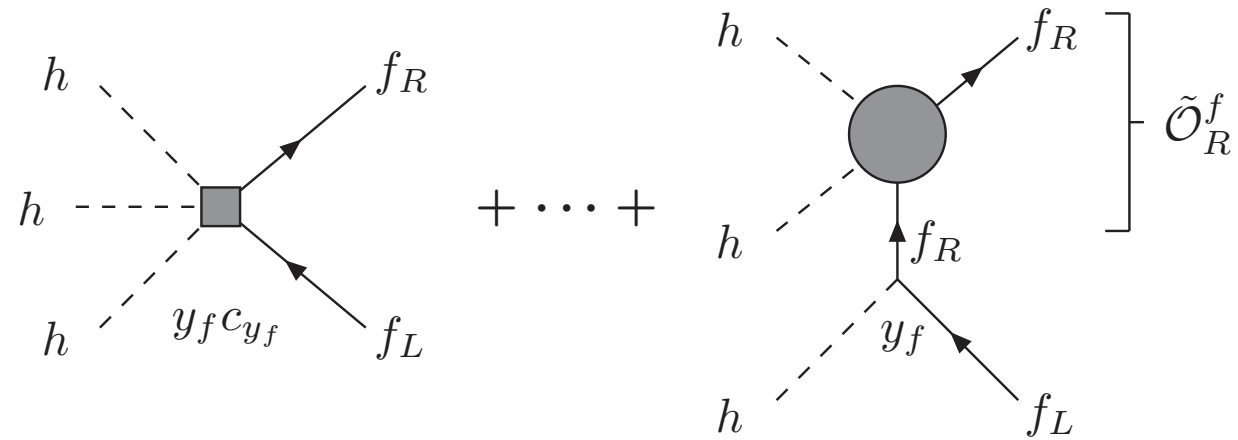

Figure 4: Contributions to the process $h$ hh $\rightarrow \bar{f}_{L} f_{R}$ at order $1 / \Lambda^{2}$, including the one-loop corrections shown in Fig. 3.

are neglecting. Notice that in the above results we have included some dependence on Wilson coefficients beyond those of Eq. (53) and $c_{r}$. In particular, we have kept the contributions from wave function renormalization (which are trivial to take into account) in all cases, and we also kept the contributions from $c_{T}$ in $\gamma_{c_{W}}$ and $\gamma_{c_{B}}$ that were already calculated in [7]. These anomalous dimensions have been calculated through the (divergent pieces of the) one-loop effective action.

Using (B.10)-(B.32) we can calculate the anomalous dimensions $\gamma_{C_{i}}$ 's for the shift-invariant Wilson coefficients. These are given in Section 5. We have cross-checked those RGEs by calculating them in an alternative way. We have looked at the one-loop radiative corrections to some particular physical processes and required the corresponding amplitudes to be independent of the renormalization scale. In order to find agreement between both methods, it is crucial to include in the amplitude for the physical process non-1PI contributions. In the effective action approach, such diagrams are in one-to-one correspondence with the redundant operators being eliminated.

As an illustrative example of the previous point, consider the contribution of the redundant operator $\tilde{\mathcal{O}}_{R}^{f}$ to the renormalization of $\mathcal{O}_{y_{f}}$. One-loop radiative corrections do generate $\tilde{\mathcal{O}}_{R}^{f}$ in the one-loop effective action, as shown in Fig. 3, even if we remove $\tilde{\mathcal{O}}_{R}^{f}$ from the (tree-level) Lagrangian. The physical combination $C_{y_{f}}$ [see Eq. (B.7)] depends on $\tilde{c}_{R}^{f}$ and, therefore, the 
anomalous dimension $\gamma_{C_{y_{f}}}$ also depends on $\gamma_{\tilde{c}_{R}^{f}}$. The same result for $\gamma_{{y_{f}}_{f}}$ can be obtained by looking at the physical process $h h h \rightarrow \bar{f}_{L} f_{R}$. The $1 / \Lambda^{2}$ diagrammatic contributions to this process are shown in Fig. 4. Besides the tree-level contribution through $c_{y_{f}}$ shown on the left, there are one-loop corrections, among which we just show the ones related to the redundant operator $\tilde{\mathcal{O}}_{R}^{f}$. Having removed the redundant $\tilde{\mathcal{O}}_{R}^{f}$ from the basis, there is no treelevel contribution from $\tilde{c}_{R}^{f}$ to $h h h \rightarrow \bar{f}_{L} f_{R}$ and the divergences from the one-loop blob shown in Fig. 4 have to be absorbed by $c_{y_{f}}(\mu)$ to obtain an amplitude that is independent of the renormalization scale $\mu$.

Finally, the reader can check, using the previous anomalous dimensions which include the dependence on the redundant coefficient $c_{r}$, that the anomalous dimensions of the shift invariant combinations $c_{H}-c_{r}, c_{6}+2 c_{r}, c_{y_{t}}+c_{r} / 2, c_{y_{b}}+c_{r} / 2$, plus all the other Wilson coefficients, are functions of these same combinations, so that one can take $c_{r}=0$ in a consistent way. 


\section{Transformation properties of the $d=6$ operators under the custodial $S U(2)_{L} \otimes S U(2)_{R}$ symmetry}

The $d=6$ operators of the basis of Section 2 can be made invariant under the custodial $S U(2)_{L} \otimes S U(2)_{R}$ by promoting their coefficients to (non-propagating) spurion fields transforming under this symmetry. In this Appendix we present these transformation rules.

The bosonic sector of the SM Lagrangian can be made custodial invariant by promoting the gauge coupling $g^{\prime}$ to transform as a triplet under $S U(2)_{R}$ :

$$
g_{a}^{\prime} \sigma_{a} \rightarrow g_{a}^{\prime} R \sigma_{a} R^{\dagger}
$$

whose nonzero VEVs, given by $\left\langle g_{a}^{\prime}\right\rangle=g^{\prime} \delta_{a, 3}$, define how the custodial symmetry is explicitly broken by this coupling. For the Higgs field, that transforms as a $\left(\mathbf{2}_{L}, \mathbf{2}_{R}\right)$, it is convenient to use the matrix field

$$
\Sigma=\frac{1}{\sqrt{2}}(\tilde{H}, H)
$$

that transforms under the custodial group as $\Sigma \rightarrow L \Sigma R^{\dagger}$, and therefore its covariant derivative is given by $D_{\mu} \Sigma=\partial_{\mu} \Sigma-i g W_{L \mu}^{a} \sigma^{a} \Sigma / 2+i g^{\prime a} B_{\mu} \Sigma \sigma^{a} / 2$.

To write the Yukawa sector of the SM invariant under the custodial symmetry, we can promote the Yukawa couplings to transform as a doublet under $S U(2)_{R}$ :

$$
Y_{u} \rightarrow R Y_{u}
$$

where $\left\langle Y_{u}\right\rangle=\left(y_{u}, 0\right)^{T}$, and similarly for the other Yukawas. The Yukawa term is then written as $\sqrt{2} \bar{Q}_{L} \Sigma Y_{u} u_{R}$, where the SM fermions transform as singlets under $S U(2)_{R}$. To define the proper hypercharge assignment for the SM fermions, we have to enlarge the global group to $S U(2)_{L} \otimes S U(2)_{R} \otimes U(1)_{X}$ and define the hypercharge as $Y=T_{R}^{3}+X$. This means that the $U(1)_{Y}$ is not only contained in $S U(2)_{R}$ but also in $U(1)_{X}$ and therefore $g^{\prime}$ has also a singlet component under the custodial group.

Using the above definitions we can write the Lagrangian $\mathcal{L}_{6}$ as an invariant under $S U(2)_{L} \otimes$ $S U(2)_{R}$. This requires to promote few of the coefficients to spurion fields transforming nontrivially. The result is shown in Table 4 . Only $c_{T}$ and $c_{L, R}$ transform non-trivially, being then, together with $g^{\prime}$ and the Yukawa couplings, the only sources of custodial breaking. This information is useful to deduce what combinations of coefficients and couplings can contribute at the one-loop level to a given anomalous dimension. For example, contributions to $\gamma_{c_{T}}$ can only come from terms that transform as $\mathbf{5}_{R}:\left\langle g_{a}^{\prime} g_{b}^{\prime} c_{H}\right\rangle=g^{2} c_{H} \delta_{a, 3} \delta_{b, 3}$ and $\left\langle Y_{u}^{\dagger} \sigma^{a} Y_{u} c_{L, R}^{b}\right\rangle=$ $-y_{u}^{2} c_{L, R} \delta^{a, 3} \delta^{b, 3}$, as the explicit calculation shows. In the same way it can be understood why $\gamma_{c_{H}}$ depends on $y_{t}^{2} c_{L}^{(3)}$ but not on $y_{t}^{2} c_{L}$, being $c_{H}$ a singlet under the custodial symmetry.

Useful information can also be derived from the transformations under the parity $P_{L R}$ that interchanges $L \leftrightarrow R$. In the bosonic sector, we have

$$
\Sigma \leftrightarrow \Sigma^{\dagger}
$$




$$
\begin{aligned}
\frac{g^{\prime a}}{g^{\prime}} B_{\mu} & \leftrightarrow W_{L \mu}^{a} \\
g^{\prime} & \leftrightarrow g \\
c_{H} & \leftrightarrow c_{H} \\
c_{W} & \leftrightarrow c_{B} \\
\kappa_{H W} & \leftrightarrow \kappa_{H B},
\end{aligned}
$$

while $c_{T}$ and $\kappa_{B B}$ do not have a well-defined transformation property inside the operator basis. For this reason it could be convenient to work with the operator $\mathcal{O}_{W B}$ instead of $\mathcal{O}_{B B}$ [both related by Eq. (13)] that is even under $P_{L R}$, and therefore $\kappa_{W B} \leftrightarrow \kappa_{W B}$.

For operators involving SM fermions, we have several possibilities for the transformation properties under $P_{L R}$, see [33]. The two simplest ones are to consider (for the up-type quark)

$$
\text { I) } Q_{R} \equiv \frac{1}{y_{u}} Y_{u} u_{R} \quad \text { and } \quad Q_{L}
$$

that transform respectively as $\left(\mathbf{1}, \mathbf{2}_{R}\right)_{1 / 6}$ and $\left(\mathbf{2}_{L}, \mathbf{1}\right)_{1 / 6}$ under $S U(2)_{L} \otimes S U(2)_{R} \otimes U(1)_{X}$, or, alternatively,

$$
\text { II) } \mathcal{Q}_{L} \equiv \frac{1}{y_{u}} Q_{L} \otimes Y_{u}^{\dagger} \quad \text { and } \quad u_{R}
$$

transforming as $\left(\mathbf{2}_{L}, \mathbf{2}_{R}\right)_{2 / 3}$ and $(\mathbf{1}, \mathbf{1})_{2 / 3}$ respectively. For the first case, Eq. (C.5), we can write the operators $\mathcal{O}_{R}$ and $\mathcal{O}_{L}^{(3)}$ in the following way:

$$
-i c_{R} \operatorname{tr}\left[\sigma_{a} \Sigma^{\dagger} \stackrel{\leftrightarrow}{D}_{\mu} \Sigma\right] \bar{Q}_{R} \sigma_{a} \gamma^{\mu} Q_{R} \text { and } i c_{L}^{(3)} \operatorname{tr}\left[\Sigma^{\dagger} \sigma_{a} \stackrel{\leftrightarrow}{D}_{\mu} \Sigma\right] \bar{Q}_{L} \sigma_{a} \gamma^{\mu} Q_{L}
$$

such that under $P_{L R}$ we can define $Q_{L} \leftrightarrow Q_{R}$ and

$$
\text { I) } c_{R} \leftrightarrow c_{L}^{(3)}
$$

For the second case, Eq. (C.6), we can write the operators $\mathcal{O}_{L}$ and $\mathcal{O}_{L}^{(3)}$ as

$$
i c_{L} \operatorname{tr}\left[\sigma_{a} \Sigma^{\dagger} \stackrel{\leftrightarrow}{D}_{\mu} \Sigma\right] \operatorname{tr}\left[\overline{\mathcal{Q}}_{L}^{T} \epsilon^{T} \sigma_{a} \gamma^{\mu} \epsilon \mathcal{Q}_{L}^{T}\right] \text { and } i c_{L}^{(3)} \operatorname{tr}\left[\Sigma^{\dagger} \sigma_{a} \stackrel{\leftrightarrow}{D}_{\mu} \Sigma\right] \operatorname{tr}\left[\overline{\mathcal{Q}}_{L} \sigma_{a} \gamma^{\mu} \mathcal{Q}_{L}\right]
$$

and define $\mathcal{Q}_{L} \leftrightarrow \epsilon \mathcal{Q}_{L}^{T} \epsilon^{T}$ under $P_{L R}$ that gives the transformation rule

$$
\text { II) } c_{L} \leftrightarrow-c_{L}^{(3)}
$$

In this latter case, invariance under $P_{L R}$ implies $c_{L}+c_{L}^{(3)}=0$, and therefore no corrections to the $Z b_{L} \bar{b}_{L}$ coupling. 


\begin{tabular}{|c|c|c|c|}
\hline Operator & Spurion & $S U(2)_{L} \otimes S U(2)_{R}$ & VEV \\
\hline$\frac{1}{2} c_{T}^{a, b} \operatorname{tr}\left[\sigma^{a} \Sigma^{\dagger} \stackrel{\leftrightarrow}{D}_{\mu} \Sigma\right] \operatorname{tr}\left[\sigma^{b} \Sigma^{\dagger} \stackrel{\leftrightarrow}{D}^{\mu} \Sigma\right]$ & $c_{T}^{a, b}$ & $\left(\mathbf{3}_{R} \otimes \mathbf{3}_{R}\right)_{s}=\mathbf{5}_{R}+\mathbf{1}$ & $c_{T} \delta^{a, 3} \delta^{b, 3}$ \\
\hline$\frac{1}{2} c_{H}\left(\partial_{\mu} \operatorname{tr}\left[\Sigma^{\dagger} \Sigma\right]\right)^{2}$ & $c_{H}$ & 1 & $c_{H}$ \\
\hline$c_{6}\left(\operatorname{tr}\left[\Sigma^{\dagger} \Sigma\right]\right)^{3}$ & $c_{6}$ & 1 & $c_{6}$ \\
\hline$-\frac{i}{2} c_{B} g^{\prime a} \operatorname{tr}\left[\sigma^{a} \Sigma^{\dagger} \stackrel{\leftrightarrow}{D}_{\mu} \Sigma\right] \partial_{\nu} B^{\mu \nu}$ & $c_{B}$ & 1 & $c_{B}$ \\
\hline$\frac{i}{2} c_{W} g \operatorname{tr}\left[\Sigma^{\dagger} \sigma_{a} \stackrel{\leftrightarrow}{D}_{\mu} \Sigma\right] D_{\nu} W_{a}^{\mu \nu}$ & $c_{W}$ & 1 & $c_{W}$ \\
\hline$c_{y_{u}} \operatorname{tr}\left[\Sigma^{\dagger} \Sigma\right] \sqrt{2} \bar{Q}_{L} \Sigma Y_{u} u_{R}$ & $c_{y_{u}}$ & 1 & $c_{y_{u}}$ \\
\hline$-i c_{R}^{a} \operatorname{tr}\left[\sigma^{a} \Sigma^{\dagger} \stackrel{\leftrightarrow}{D}_{\mu} \Sigma\right] \bar{f}_{R} \gamma^{\mu} f_{R}$ & $c_{R}^{a}$ & $\mathbf{3}_{R}$ & $c_{R} \delta^{a, 3}$ \\
\hline$-i c_{L}^{a} \operatorname{tr}\left[\sigma^{a} \Sigma^{\dagger} \stackrel{\leftrightarrow}{D}_{\mu} \Sigma\right] \bar{f}_{L} \gamma^{\mu} f_{L}$ & $c_{L}^{a}$ & $\mathbf{3}_{R}$ & $c_{L} \delta^{a, 3}$ \\
\hline$i c_{L}^{(3)} \operatorname{tr}\left[\Sigma^{\dagger} \sigma_{a} \stackrel{\leftrightarrow}{D}_{\mu} \Sigma\right] \bar{f}_{L} \sigma_{a} \gamma^{\mu} f_{L}$ & $c_{L}^{(3)}$ & 1 & $c_{L}^{(3)}$ \\
\hline$-4 i c_{R}^{u d} \operatorname{tr}\left[\Sigma Y_{d} Y_{u}^{\dagger} D_{\mu} \Sigma^{\dagger}\right] \bar{u}_{R} \gamma^{\mu} d_{R}$ & $c_{R}^{u d}$ & 1 & $c_{R}^{u d}$ \\
\hline$\kappa_{B B} g^{\prime a} g^{\prime a} \operatorname{tr}\left[\Sigma^{\dagger} \Sigma\right] B_{\mu \nu} B^{\mu \nu}$ & $\kappa_{B B}$ & 1 & $\kappa_{B B}$ \\
\hline$-i \kappa_{H B} g^{\prime a} \operatorname{tr}\left[\sigma^{a} D_{\mu} \Sigma^{\dagger} D_{\nu} \Sigma\right] B^{\mu \nu}$ & $\kappa_{H B}$ & 1 & $\kappa_{H B}$ \\
\hline$i \kappa_{H W} g \operatorname{tr}\left[D_{\mu} \Sigma^{\dagger} \sigma_{a} D_{\nu} \Sigma\right] W_{a}^{\mu \nu}$ & $\kappa_{H W}$ & 1 & $\kappa_{H W}$ \\
\hline$\kappa_{D B} \sqrt{2} \bar{Q}_{L} \Sigma Y_{u} \sigma^{\mu \nu} u_{R} B_{\mu \nu}$ & $\kappa_{D B}$ & 1 & $\kappa_{D B}$ \\
\hline$\kappa_{D W} \sqrt{2} \bar{Q}_{L} \sigma^{a} \Sigma Y_{u} \sigma^{\mu \nu} u_{R} W_{\mu \nu}^{a}$ & $\kappa_{D W}$ & 1 & $\kappa_{D W}$ \\
\hline$\kappa_{D G} \sqrt{2} \bar{Q}_{L} \Sigma Y_{u} T^{A} \sigma^{\mu \nu} u_{R} G_{\mu \nu}^{A}$ & $\kappa_{D G}$ & 1 & $\kappa_{D G}$ \\
\hline
\end{tabular}

Table 4: Transformation of the spurion Wilson coefficients of the $d=6$ operators under the custodial symmetry and their corresponding VEV. We are dropping fermion indices in the coefficients. 


\section{References}

[1] S. Chatrchyan et al. [CMS Collab.], Phys. Lett. B 710 (2012) 403 [hep-ex/1202.1487]; G. Aad et al. [ATLAS Collab.], Phys. Rev. Lett. 108 (2012) 111803 [hep-ex/1202.1414].

[2] W. Buchmüller and D. Wyler, Nucl. Phys. B 268 (1986) 621.

[3] A. Pomarol and F. Riva, [hep-ph/1308.2803].

[4] G. F. Giudice, C. Grojean, A. Pomarol and R. Rattazzi, JHEP 0706 (2007) 045 [hep$\mathrm{ph} / 0703164]$.

[5] R. Contino, M. Ghezzi, C. Grojean, M. Mühlleitner and M. Spira, [hep-ph/1303.3876].

[6] C. Grojean, E. E. Jenkins, A. V. Manohar and M. Trott, JHEP 1304 (2013) 016 [hep$\mathrm{ph} / 1301.2588]$.

[7] J. Elias-Miró, J. R. Espinosa, E. Masso and A. Pomarol, [hep-ph/1302.5661].

[8] K. Hagiwara, S. Ishihara, R. Szalapski and D. Zeppenfeld, Phys. Lett. B 283 (1992) 353; Phys. Rev. D 48 (1993) 2182.

[9] B. Grzadkowski, M. Iskrzynski, M. Misiak and J. Rosiek, JHEP 1010 (2010) 085 [hep$\mathrm{ph} / 1008.4884]$.

[10] C. Arzt, M. B. Einhorn and J. Wudka, Nucl. Phys. B 433 (1995) 41 [hep-ph/9405214].

[11] J. A. Aguilar-Saavedra, Nucl. Phys. B 843 (2011) 638 [Erratum-ibid. B 851 (2011) 443].

[12] G. D'Ambrosio, G. F. Giudice, G. Isidori and A. Strumia, Nucl. Phys. B 645 (2002) 155 [hep-ph/0207036], and references therein.

[13] O. Domenech, A. Pomarol and J. Serra, Phys. Rev. D 85 (2012) 074030 [hep$\mathrm{ph} / 1201.6510]$.

[14] M. E. Peskin and T. Takeuchi, Phys. Rev. Lett. 65 (1990) 964.

[15] R. Barbieri, A. Pomarol, R. Rattazzi and A. Strumia, Nucl. Phys. B 703 (2004) 127 [hep-ph/0405040].

[16] M. Farina, C. Grojean, F. Maltoni, E. Salvioni and A. Thamm, JHEP 1305 (2013) 022 [hep-ph/1211.3736].

[17] A. De Rújula, M. B. Gavela, P. Hernández and E. Masso, Nucl. Phys. B 384 (1992) 3.

[18] See for example, A. Falkowski, F. Riva and A. Urbano, [hep-ph/1303.1812].

[19] The LEP collaborations ALEPH, DELPHI, L3, OPAL, and the LEP TGC Working Group, LEPEWWG/TGC/2003-01. 
[20] G. Aad et al. [ATLAS Collab.], Phys. Rev. D 87 (2013) 112001 [hep-ex/1210.2979]; S. Chatrchyan et al. [CMS Collaboration], [hep-ex/1306.1126].

[21] For reviews, see, for example, V. Lombardo [on behalf of the ATLAS and CMS Collab.], talk at QCD Moriond 2013 [hep-ex/1305.3773], and J. Stark, talk at EPS HEP 2013 conference, Stokholm, July 2013.

[22] M. Antonelli, V. Cirigliano, G. Isidori, F. Mescia, M. Moulson, H. Neufeld, E. Passemar and M. Palutan et al., Eur. Phys. J. C 69 (2010) 399 [hep-ph/1005.2323].

[23] S. Chatrchyan et al. [CMS Collaboration], Phys. Rev. D 87 (2013) 072005 [hepex/1302.2812].

[24] ATLAS Collab., ATLAS-CONF-2013-033; CMS Collab., CMS-PAS-TOP-12-025.

[25] ATLAS Collab., ATLAS-CONF-2012-126, CMS Collab., PAPER-TOP-12-014.

[26] N. Vignaroli, Phys. Rev. D 86 (2012) 115011 [hep-ph/1204.0478].

[27] T. Corbett, O. J. P. Éboli, J. González-Fraile and M. C. González-García, Phys. Rev. D 87 (2013) 015022 [hep-ph/1211.4580].

[28] S. Alam, S. Dawson and R. Szalapski, Phys. Rev. D 57 (1998) 1577 [hep-ph/9706542].

[29] R. Barbieri, B. Bellazzini, V. S. Rychkov and A. Varagnolo, Phys. Rev. D 76 (2007) 115008 [hep-ph/0706.0432].

[30] A. Pomarol and J. Serra, Phys. Rev. D 78 (2008) 074026 [hep-ph/0806.3247].

[31] See, for example, R. S. Gupta, M. Montull and F. Riva, JHEP 1304 (2013) 132 [hep$\mathrm{ph} / 1212.5240]$.

[32] M. B. Einhorn and J. Wudka, JHEP 0108 (2001) 025 [hep-ph/0105035].

[33] K. Agashe, R. Contino, L. Da Rold and A. Pomarol, Phys. Lett. B 641 (2006) 62 [hep$\mathrm{ph} / 0605341]$. 